TRANSACTIONS OF THE

AMERICAN MATHEMATICAL SOCIETY

Volume 349, Number 3, March 1997, Pages 1029-1062

S 0002-9947(97)01854-0

\title{
THE MIZOHATA COMPLEX
}

\author{
ABDELHAMID MEZIANI
}

\begin{abstract}
This paper deals with the local solvability of systems of first order linear partial differential equations defined by a germ $\omega$ at $0 \in \mathbb{R}^{n+1}$ of a $\mathbb{C}$ valued, formally integrable $(\omega \wedge d \omega=0)$, 1-form with nondegenerate Levi form. More precisely, the size of the obstruction to the solvability, for $(q-1)$-forms $u$, of the equation

$$
d u \wedge \omega=\eta \wedge \omega
$$

where $\eta$ is a given $q$-form satisfying $d \eta \wedge \omega=0$ is estimated in terms of the De Rham cohomology relative to $\omega$
\end{abstract}

\section{INTRODUCTION}

This paper deals with the obstruction to the local solvability problem of a formally integrable form (or equivalently of a system of vector fields). More precisely, let $\omega$ be the germ at $0 \in \mathbb{R}^{n+1}$ of a $\mathbb{C}$-valued smooth 1 -form satisfying

$$
\omega(0) \neq 0 \text { and } \quad d \omega \wedge \omega=0 .
$$

Two smooth germs $\eta$ and $\eta^{\prime}$ of $q$-forms are said to be $\omega$-equivalent if $\eta^{\prime}=\eta+\alpha \wedge \omega$ for some $(q-1)$-form $\alpha$. Denote by $\Omega_{\omega}^{q}$ the quotient space, i.e. $\Omega_{\omega}^{q}=\Omega^{q} / \Omega^{q-1} \wedge \omega$, where $\Omega^{q}$ is the space of germs of $q$-forms. It follows from (1) that the exterior derivative $d: \Omega^{q} \longrightarrow \Omega^{q+1}$ induces a complex

$$
0 \longrightarrow \Omega^{0} \longrightarrow \Omega_{\omega}^{1} \longrightarrow \cdots \longrightarrow \Omega_{\omega}^{n} \longrightarrow 0
$$

whose cohomology we denote by $H_{\omega}^{*}$. It is the De Rham cohomology relative to $\omega$. Notice that $H_{\omega}^{0}$ is the space of first integrals of $\omega$, which is reduced to $\mathbb{C}$ when $\omega$ is without integrating factor. The only two situations in which this complex is fully understood are the real case $(\omega \wedge \bar{\omega} \equiv 0)$ and the elliptic case $(\omega \wedge \bar{\omega} \neq 0)$. In both cases the cohomology groups $H_{\omega}^{q}$ are trivial when $q \geq 1$. The planar case of a real analytic form $\omega$ has been studied in [Me1]. Otherwise the focus has been, so far, in finding conditions for the triviality of $H_{\omega}^{q}$, when

$$
\omega=d(x+i \phi(x, t)) \text {. }
$$

This was initiated by Nirenberg and Treves in $[\mathrm{NT}]$. The tube case $(\phi$ independent on $x$ ) has been solved by Treves in [Tr1] who proved that the triviality of the $(q-1)$-th cohomology of the level sets of $\phi$ is equivalent to the triviality of $H_{\omega}^{q}$. Among results in this direction (when $\phi$ depends effectively on $x$ ), we would like to mention $[\mathrm{CH}],[\mathrm{CT}],[\mathrm{MT}],[\mathrm{Tr} 2],[\mathrm{Tr} 3]$. In particular, it is proved in $[\mathrm{CT}]$ that the nontriviality of the $(q-1)$-th cohomology of the level set of $x+i \phi$ implies the

Received by the editors August 12, 1994 and, in revised form, June 27, 1995.

1991 Mathematics Subject Classification. Primary 35F05; Secondary 58A10.

(C)1997 American Mathematical Society 
nontriviality of $H_{\omega}^{q}$. The converse of this (which has been proved to hold when $q=1$, [Tr2] and [MT], and in top forms [Tr3]) has been conjectured by Treves.

In this paper we give a complete description of $H_{\omega}^{*}$ when the form $\omega$ defines a Mizohata structure at $0 \in \mathbb{R}^{n+1}$ (this means that $\omega$ has nondegenerate Levi form at 0 ). Our primary motivation for investigating such structures (besides the fact that they appear next to the elliptic ones in the hierarchy of complexity) is that these are the generic ones among all nonelliptic structures. Our basic idea here is in the viewing of the Mizohata form $\omega$ as defining a fibration in each component of the complement of the separatrix, $V=\{q(t)=0\}$ (defined below by (4)), of the foliation defined by $\omega$. More precisely, if $W_{i}$ is a component of $\mathbb{R}^{n+1} \backslash V, L_{i}$ the generic fiber of $\omega$ in $W_{i}$, and $z_{i}$ a first integral of $\omega$ in $W_{i}$, then $W_{i}$ is diffeomorphic to $L_{i} \times z_{i}\left(W_{i}\right)$. Hence the cohomology relative to $\omega$ in $W_{i}$ can be expressed in terms of the cohomology relative to $d z_{i}$ in the product manifold $L_{i} \times z_{i}\left(W_{i}\right)$. The passage from the different components $W_{i}$ 's to a full neighborhood of $0 \in \mathbb{R}^{n+1}$ is done via a reduction to forms that are flat along $V$. This in turn is achieved thanks to Borel's extension theorem and the formal solvability of $\omega$.

When $\omega$ defines a Mizohata structure, we can find coordinates $\left(x, t_{1}, \cdots, t_{n}\right)$ in $\mathbb{R}^{n+1}$ (see $[\operatorname{Tr} 4]$ ) such that $\omega$ can be written as

$$
\omega=d(x+i q(t))+\sum_{j=1}^{n} a_{j}(x, t) d t_{j},
$$

where

$$
q(t)=t_{1}^{2}+\cdots+t_{k}^{2}-t_{k+1}^{2}-\cdots-t_{n}^{2}
$$

and the $a_{j}$ 's are flat along $t=0$. The signature of such structure is the number $\nu=|n-2 k|$. When $\nu \neq|n-2|$, it has been proved ([Me2]) that $\omega$ has an integrating factor and thus can be written as

$$
\omega=d(x+i q(t)) .
$$

Otherwise (when $\nu=|n-2|$ ), germs at $0 \in \mathbb{R}^{2}$ of Mizohata structures can be used to give canonical representation for $\omega$. More precisely, if $\nu=|n-2|$ and $n>2$, then $\omega$ can be written as

$$
\begin{array}{ll}
\omega=d x+i(1+\rho(x, \sqrt{q(t)})) d q(t) & \text { in }\{q(t) \geq 0\} \cap\left\{t_{1} \geq 0\right\}, \\
\omega=d(x+i q(t)) & \text { in }\{q(t) \leq 0\} \cup\left\{t_{1} \geq 0\right\},
\end{array}
$$

where $\rho(x, s)$ is a smooth function in the $(x, s)$-plane with support in $s \geq 0$. Hence, $\omega$ is completely determined by the two-dimensional Mizohata structure

$$
\omega_{\rho}^{0}=d x+i(1+\rho(x, s)) d s^{2} .
$$

When $\nu=0$ and $n=2$, then $\omega$ can be written as

$$
\begin{array}{ll}
\omega=d x+i\left(1+\rho_{1}\left(x, \sqrt{t_{1}^{2}-t_{2}^{2}}\right)\right) d\left(t_{1}^{2}-t_{2}^{2}\right) & \text { in }\left\{t_{1}^{2}-t_{2}^{2} \geq 0\right\} \cap\left\{t_{1} \geq 0\right\}, \\
\omega=d x+i\left(1+\rho_{2}\left(x, \sqrt{t_{2}^{2}-t_{1}^{2}}\right)\right) d\left(t_{1}^{2}-t_{2}^{2}\right) & \text { in }\left\{t_{1}^{2}-t_{2}^{2} \leq 0\right\} \cap\left\{t_{2} \geq 0\right\}, \\
\omega=d\left(x+i\left(t_{1}^{2}-t_{2}^{2}\right)\right) & \text { elsewhere, }
\end{array}
$$

where $\rho_{1}$ and $\rho_{2}$ are smooth functions in the $(x, s)$-plane with support in $s \geq 0$. Here $\omega$ is determined by the two two-dimensional Mizohata structures

$$
\omega_{\rho_{1}}^{0}=d x+i\left(1+\rho_{1}\right) d s^{2} \quad \text { and } \quad \omega_{\rho_{2}}^{0}=d x-i\left(1+\rho_{2}\right) d s^{2} .
$$


One of the main results of this paper (theorem 11.1) is that for $q \geq 2$,

$$
\begin{array}{ll}
H_{\omega}^{q} \cong H^{q-1}\left(S^{k-1} \times S^{n-k-1}\right) \otimes \mathcal{S} & \text { if }|n-2 k| \neq n ; \\
H_{\omega}^{q} \cong H^{q-1}\left(S^{n-1}\right) \otimes \mathcal{S} & \text { if }|n-2 k|=n,
\end{array}
$$

where $\omega$ is the germ of a Mizohata structure with signature $|n-2 k|, S^{m}$ is the $m$ sphere, $H^{*}(M)$ is the De-Rham cohomology of the manifold $M$, and where

$$
\mathcal{S}=H_{d(x+i y)}^{1}\left(\mathcal{F}_{0}(\{y=0\})\right)
$$

(see notation in section 1). Notice that $H_{\omega}^{1}$ is trivial when $\nu \neq|n-2|$ (this results from (5) and [Tr1]).

The study of $H_{\omega}^{1}$ for the critical signature $\nu=|n-2|$ necessitates the introduction of new spaces built from the spaces $\mathcal{A}_{+}$and $\mathcal{A}_{-}$of germs at $0 \in \mathbb{R}$ of $C^{\infty}$ functions that have a holomorphic extension to the upper and lower half plane, respectively. These new spaces are built as follows. For a germ $l$ of an increasing diffeomorphism at $0 \in \mathbb{R}$, consider the equivalence relation in $\mathcal{A}_{-} \times \mathcal{A}_{-}$given by

$$
(\alpha, \beta) \sim\left(\alpha^{\prime}, \beta^{\prime}\right) \Longleftrightarrow\left\{\begin{array}{c}
\exists(h, k) \in \mathcal{A}_{+} \times \mathcal{A}_{+} ; \\
\left(\alpha-\alpha^{\prime}\right) \circ l+h \circ l=\left(\beta-\beta^{\prime}\right)+k .
\end{array}\right.
$$

Let $\mathcal{Y}_{l}$ denote the quotient space. If $\omega$ is the germ at $0 \in \mathbb{R}^{2}$ of a Mizohata structure and the class of $l$ in the space of Sjöstrand invariants (see [Tr4]) is a representative of $\omega$, we prove (Theorem 12.1) that

$$
H_{\omega}^{1} \cong \mathcal{Y}_{l}
$$

For $l$ as above, define an equivalence relation in $\mathcal{A}_{-} \times \mathcal{A}_{-} \times \mathcal{A}_{+}$by

$$
(\alpha, \beta, \gamma) \sim\left(\alpha^{\prime}, \beta^{\prime}, \gamma^{\prime}\right) \Longleftrightarrow\left\{\begin{aligned}
\exists(h, k, m) \in \mathcal{A}_{+} & \times \mathcal{A}_{+} \times \mathcal{A}_{-} ; \\
\left(\alpha-\alpha^{\prime}\right) \circ l+h \circ l & =\left(\beta-\beta^{\prime}\right)+k \\
& =\left(\gamma-\gamma^{\prime}\right)+m .
\end{aligned}\right.
$$

If $\mathcal{Z}_{l}$ is the quotient space, $\omega$ is as in (6), and $l$ is a representative of $\omega_{\rho}^{0}$ given in (7), then we prove (Theorem 13.1) that

$$
H_{\omega}^{1} \cong \mathcal{Z}_{l} .
$$

Finally, for the case $\nu=0$ and $n=2$ and $\omega$ as in (8), we need two germs $l_{1}$ and $l_{2}$ representing $\omega_{\rho_{1}}^{0}$ and $\omega_{\rho_{2}}^{0}$ of (9). For such germs we define a relation in $\mathcal{A}_{-} \times \mathcal{A}_{-} \times \mathcal{A}_{+} \times \mathcal{A}_{+}$by

$$
(\alpha, \beta, \gamma, \delta) \sim\left(\alpha^{\prime}, \beta^{\prime}, \gamma^{\prime}, \delta^{\prime}\right) \Longleftrightarrow\left\{\begin{aligned}
\exists(h, k, m, n) \in \mathcal{A}_{+} & \times \mathcal{A}_{+} \times \mathcal{A}_{-} \times \mathcal{A}_{-} ; \\
\left(\alpha-\alpha^{\prime}\right) \circ l_{1}+h \circ l_{1} & =\left(\beta-\beta^{\prime}\right)+k \\
& =\left(\gamma-\gamma^{\prime}\right) \circ l_{2}+m \circ l_{2} \\
& =\left(\delta-\delta^{\prime}\right)+n .
\end{aligned}\right.
$$

If $\mathcal{W}_{l_{1}, l_{2}}$ is the quotient space, then we prove (Theorem 14.1) that

$$
H_{\omega}^{1} \cong \mathcal{W}_{l_{1}, l_{2}} .
$$

The organization of this paper is as follows. Sections 1 to 5 are devoted to definitions and preparatory results. Mizohata structures as well as their classification according to [Me2] are recalled in sections 6 and 7 . The reduction to flatness along the variety $V$ is achieved in sections 8 and 9 . Section 10 deals with the cohomology 
with support in a component of $\mathbb{R}^{n+1} \backslash V$. In sections $11,12,13$, and 14 , we prove, respectively, isomorphisms (10), (11), (12) and (13). The closing section is a remark about Treves conjecture.

\section{Definitions, NOtation AND PREPARAtory RESUlts}

We set here the notation of some $\mathbb{C}$-valued function spaces that we will be dealing with, then we define the De Rham cohomology groups relative to a formally integrable form, and finally state some results whose proofs are contained in sections 2 to 5 .

Let $N$ be a $C^{\infty}$ manifold, $A \subset N$ and $p \in A$. Denote by: $\mathcal{E}(A)$ the space of germs along $A$ of $C^{\infty}$ functions on $N$;

$\mathcal{F}(A)$ the subspace of $\mathcal{E}(A)$ consisting of those germs that are flat along $A$ (a function is flat along $A$ if it vanishes along $A$ together with all its derivatives);

$\mathcal{F}_{p}(A)$ the space of germs at $p$ of functions that are flat along $A$;

$\mathcal{E}^{m}$ the space of germs at $0 \in \mathbb{R}^{m}$ of $C^{\infty}$ functions;

$\mathbb{C}\{z\}$ the ring of convergent power series in the variable $z$;

$K\left[\xi_{1}, \cdots, \xi_{m}\right]$ the ring of formal power series in $\xi_{1}, \cdots, \xi_{m}$ with coefficients in a $\operatorname{ring} K$;

$\mathcal{S}$ is the quotient space obtained from the space of germs at $0 \in \mathbb{R}^{2}$ of $C^{\infty}$ functions with compact support in the upper half plane $\{y \geq 0\}$, by identifying two functions $f$ and $f^{\prime}$ if the function of the real variable $x$,

$$
\iint_{\mathbb{C}} \frac{f(\zeta)-f^{\prime}(\zeta)}{x-\zeta} d \zeta \wedge d \bar{\zeta}
$$

is real analytic at $0 \in \mathbb{R}$.

$\mathcal{A}_{\text {- }}$ the space of germs at $0 \in \mathbb{R}$ of $C^{\infty}$ functions that have a holomorphic extension to the lower half plane $y<0$;

$\mathcal{A}_{+}$: the space of germs at $0 \in \mathbb{R}$ of $C^{\infty}$ functions that have a holomorphic extension to the upper half plane $y>0$;

$\Omega^{q}(X)$ the space of $q$-forms with coefficients in $X$.

The Taylor expansion of a function $f(x, t)$ at $t=0$ with respect to the group of variables $t=\left(t_{1}, \cdots, t_{m}\right)$ is the formal power series

$$
T_{t} f=\sum_{\nu \in \mathbb{Z}^{+m}} \frac{\partial^{\nu} f}{\partial t^{\nu}}(x, 0) \frac{t^{\nu}}{\nu !}
$$

The formal expansion of a differential form with respect to $t$ is the form obtained by expanding the coefficients with respect to $t$.

Now we explain the complex defined by a formally integrable 1-form. Let $\omega \in$ $\Omega^{1}\left(\mathcal{E}^{n+1}\right)$ be such that

$$
\omega(0) \neq 0 \quad \text { and } \quad d \omega \wedge \omega=0 .
$$

Condition (1.1) implies the existence of $\alpha \in \Omega^{1}\left(\mathcal{E}^{n+1}\right)$ such that

$$
d \omega=\alpha \wedge \omega .
$$

Notice however that, unlike the $\mathbb{R}$-valued situation, this formal integrability does not necessarily mean that $\omega$ has an integrating factor (see [Tr4] for details).

Let $X=\mathcal{E}^{n+1}$ or $X=\mathcal{F}_{0}(V)$, where $V \ni 0$ is a subset of $\mathbb{R}^{n+1}$. We say that two forms $\eta, \eta^{\prime} \in \Omega^{q}(X)$ are $\omega$-equivalent if

$$
\eta \wedge \omega=\eta^{\prime} \wedge \omega
$$


or equivalently if

$$
\eta^{\prime}=\eta+\mu \wedge \omega
$$

for some $\mu \in \Omega^{q-1}(X)$. Denote by $\Omega_{\omega}^{q}(X)$ the quotient space thus obtained, i.e.

$$
\Omega_{\omega}^{q}(X)=\frac{\Omega^{q}(X)}{\Omega^{q-1}(X) \wedge \omega} .
$$

It follows at once from (1.2) and (1.4) that

$$
d \eta^{\prime}=d \eta+\left(d \mu+(-1)^{q-1} \mu \wedge \alpha\right) \wedge \omega .
$$

Thus $d \eta$ and $d \eta^{\prime}$ are again $\omega$-equivalent if $\eta$ and $\eta^{\prime}$ are. Therefore the exterior derivative

$$
d: \Omega^{q}(X) \longrightarrow \Omega^{q+1}(X)
$$

descends to the quotient space and defines a complex

$$
0 \longrightarrow \Omega_{\omega}^{0}(X)=X \longrightarrow \cdots \longrightarrow \Omega_{\omega}^{q}(X) \longrightarrow \Omega_{\omega}^{q+1}(X) \longrightarrow \cdots \longrightarrow 0 .
$$

Denote by $H_{\omega}^{q}(X)$ the $q$-th cohomology group of this complex. Then,

$$
H_{\omega}^{q}(X)=\frac{Z_{\omega}^{q}(X)}{B_{\omega}^{q}(X)}
$$

where $Z_{\omega}^{q}(X)$ and $B_{\omega}^{q}(X)$ are, respectively, the space of $\omega$-closed and the space of $\omega$-exact $q$-forms and are given by

$$
\begin{aligned}
& Z_{\omega}^{q}(X)=\left\{\eta \in \Omega^{q}(X) ; d \eta \wedge \omega=0\right\}, \\
& B_{\omega}^{q}(X)=\left\{\eta \in \Omega^{q}(X) ; \exists \mu \in \Omega^{q-1}(X),(\eta-d \mu) \wedge \omega=0\right\} .
\end{aligned}
$$

Notice that

$$
H_{\omega}^{0}(X)=\{f \in X ; d f \wedge \omega=0\}
$$

is the space of first integrals (in $X$ ) of $\omega$ (which can be reduced to constants if $\omega$ is not locally integrable), and $H_{\omega}^{n+1}=0$ since $\Omega_{\omega}^{n+1}=0$. The object of this paper is the calculation of these cohomology groups when $\omega$ is the germ of a Mizohata structure (see sections 6 and 7 for definitions).

We continue this section by stating results (proved in subsequent sections) about some cohomology groups defined by $d(x+i y)$ on manifolds with boundary and for forms flat along the boundary. For $\epsilon$ and $\delta$ positive, let

$$
R^{+}(\epsilon, \delta)=\left\{(x, y) \in \mathbb{R}^{2} ;|x|<\epsilon, 0 \leq y<\delta\right\} .
$$

We have the following lemma

Lemma 1.1. Let $z=x+i y$ be defined in $R^{+}(\epsilon, \delta)$. Then

$$
H_{d(x+i y)}^{1}\left(\mathcal{F}_{0}(\{y=0\})\right) \cong \mathcal{S} .
$$

Let $M$ be an $m$-dimensional $C^{\infty}$ manifold with finite-dimensional De Rham cohomology $H^{*}(M)$. Let $Y$ be the submanifold of $M \times R^{+}(\epsilon, \delta)$ given by $y=0$, i.e.

$$
Y=\left\{(p, x, y) \in M \times R^{+}(\epsilon, \delta) ; y=0\right\} .
$$

Recall that $\mathcal{F}(Y)$ denotes the space of germs along $Y$ of $C^{\infty}$ functions in $M \times$ $R^{+}(\epsilon, \delta)$ that are flat along $Y$. Let

$$
z: M \times R^{+}(\epsilon, \delta) \longrightarrow \mathbb{C} ; \quad z(p,(x, y))=x+i y .
$$


Since the function $z$ can be thought of as a coordinate on $M \times R^{+}(\epsilon, \delta)$, then the exterior derivative $d$ induces a complex on

$$
\Omega_{d z}^{*}(\mathcal{F}(Y))=\frac{\Omega^{*}(\mathcal{F}(Y))}{\Omega^{*-1}(\mathcal{F}(Y)) \wedge d z} .
$$

By $H_{d z}^{*}(\mathcal{F}(Y))$ we denote the cohomology of this complex.

Proposition 1.1. For $1 \leq q \leq m$, we have

$$
H_{d z}^{q}(\mathcal{F}(Y)) \cong H^{q-1}(M) \otimes H_{d(x+i y)}^{1}\left(\mathcal{F}_{0}(\{y=0\})\right) \cong H^{q-1}(M) \otimes \mathcal{S} .
$$

For every $x_{0} \in(-\epsilon, \epsilon)$, consider the embedding

$$
j_{x_{0}}: M \longrightarrow M \times R^{+}(\epsilon, \delta) ; \quad j_{x_{0}}(p)=\left(p,\left(x_{0}, 0\right)\right) .
$$

Let $\theta_{1}, \cdots, \theta_{k} \in \Omega^{q-1}(M)$ be generators of $H^{q-1}(M)$ and let $f_{1}(x, y), \cdots, f_{k}(x, y)$ be $C^{\infty}$ functions in $\mathbb{R}^{2}$ with support in $R^{+}(\epsilon, \delta)$.

Proposition 1.2. Let

$$
\eta=\sum_{\nu=1}^{k} f_{\nu}(x, y) \theta_{\nu} \wedge d \bar{z}
$$

Suppose that

$$
\eta=d u \bmod (d z)
$$

where $u \in \Omega^{q-1}\left(C^{\infty}\left(M \times R^{+}(\epsilon, \delta)\right)\right)$ satisfies

$$
j_{x_{0}}^{*} u=0, \quad \forall x_{0} \in(-\epsilon, \epsilon) .
$$

Then

$$
f_{\nu}(x, y)=0 \quad \forall \nu=1, \cdots, k .
$$

The last result we state here is about the cohomology with formal coefficients and relative to a special form $d F$. Let $p(t)$ be a polynomial in $t=\left(t_{1}, \cdots, t_{n}\right)$ with $p(0)=0$. Consider the function $F$ in $\mathbb{R}^{n+2}$ given by

$$
F(x, t, s)=x+i(s+p(t)) .
$$

Let $\mathcal{E}_{x}$ be the space of germs at $0 \in \mathbb{R}$ of $C^{\infty}$ functions in the variable $x$. Recall that

$$
X=\mathcal{E}_{x}[t, s]
$$

is the ring of formal power series in $t, s$ variables and with coefficients in $\mathcal{E}_{x}$. The exterior differentiation on $\Omega^{*}(X)$ again induces a complex on

$$
\Omega_{d F}^{*}(X)=\frac{\Omega^{*}(X)}{\Omega^{*-1}(X) \wedge d F} .
$$

We denote by $H_{d F}^{*}(X)$ the corresponding cohomology. Then we have

Proposition 1.3. $H_{d F}^{0}(X) \cong \mathcal{E}_{x}$ and $H_{d F}^{q}(X)=0$ if $q>0$. 


\section{Proof of Lemma 1.1}

Let

$$
\eta=a(x, y) d x+b(x, y) d y
$$

where $a, b$ are $C^{\infty}$ functions with support in the upper half plane $y \geq 0$. The equation

$$
d u \wedge d(x+i y)=\eta \wedge d(x+i y),
$$

for the unknown function $u$ flat along $y=0$, is equivalent to the inhomogeneous CR equation

$$
\frac{\partial u}{\partial \bar{z}}=f
$$

with $f=i a-b$. Without loss of generality, we can assume that $f$ is 0 in the lower half plane. Since the function

$$
u_{0}(z)=\frac{1}{2 i \pi} \int_{\mathbb{C}} \frac{f(\zeta)}{z-\zeta} d \zeta \wedge d \bar{\zeta}
$$

is a $C^{\infty}$ solution of (2.3), then all $C^{\infty}$ solutions of (2.3) in $y \geq 0$ have the form

$$
u(z)=u_{0}(z)-h(z)
$$

where $h$ is holomorphic in $y>0$ and $C^{\infty}$ up to the boundary. For a function $u$ as in (2.5) to be flat along the $x$-axis, it is then necessary to have

$$
u_{0}(x+i 0)=h(x+i 0) \text {; }
$$

i.e. $u_{0}$ has to be the trace of a holomorphic function in the upper half plane. Since, in addition, $u_{0}$ is holomorphic in the lower half plane, then

$$
u_{0}(x)=\frac{1}{2 i \pi} \int_{\mathbb{C}} \frac{f(\zeta)}{x-\zeta} d \zeta \wedge d \bar{\zeta}
$$

is real analytic. The correspondence $\Psi$ which to a germ $\eta$ associates the equivalence class of $f$ in $\mathcal{S}$ depends only on the cohomology class of $\eta$ and thus defines an injective map from $H_{d z}^{1}(\mathcal{F}(\{y=0\}))$ into $\mathcal{S}$. To show that this map is also surjective, it is enough to notice that if $f$ is a representative of an element in $\mathcal{S}$, then $\Psi$ maps the cohomology class of $-i f(x, y) d x$ to the class of $f$. This proves the lemma

\section{Proof of Proposition 1.1}

We start by setting notation. If $\alpha$ is a $q$-form on $M$ depending on parameter $z$, then we will denote by $\alpha_{\bar{z}}$ the $q$-form on $M$ obtained from $\alpha$ by differentiating the coefficients with respect to $\bar{z}=x-i y$. More precisely, if in local coordinates $\tau=\left(\tau_{1}, \cdots, \tau_{m}\right)$ of $M$

$$
\alpha=\sum_{|J|=q} \alpha_{J}(\tau, z) d \tau_{J},
$$

then

$$
\alpha_{\bar{z}}=\sum_{|J|=q} \frac{\partial \alpha_{J}}{\partial \bar{z}}(\tau, z) d \tau_{J} .
$$

Let $\eta$ be a $q$-form in $M \times R^{+}(\epsilon, \delta)$. We can write

$$
\eta=\eta^{0}+\eta^{1} \wedge d \bar{z} \bmod (d z),
$$


where $\eta^{0}$ and $\eta^{1}$ are, respectively, $q$ - and ( $\left.q-1\right)$-forms on $M$ depending on parameter $z$. If we denote the exterior differentiation on $M$ by $d_{0}$ and that on $M \times R^{+}(\epsilon, \delta)$ by $d$, then

$$
d \eta=d_{0} \eta^{0}+\left(d_{0} \eta^{1}+(-1)^{q} \eta_{\bar{z}}^{0}\right) \wedge d \bar{z} \quad \bmod (d z) .
$$

With this notation in mind, we start the proof. Let $\eta \in Z^{q} d z(\mathcal{F}(Y))$. Then from

$$
0=d \eta \wedge d z=d_{0} \eta^{0} \wedge d z+\left(d_{0} \eta^{1}+(-1)^{q} \eta_{\bar{z}}^{0}\right) \wedge d \bar{z} \wedge d z
$$

it follows at once that

$$
\begin{aligned}
d_{0} \eta^{0} & =0, \\
d_{0} \eta^{1}+(-1)^{q} \eta_{\bar{z}}^{0} & =0 .
\end{aligned}
$$

Let $\sigma_{1}, \cdots, \sigma_{l} \in \Omega^{q}\left(C^{\infty}(M)\right)$ be generators of $H^{q}(M)$. It follows from $\left(3.6_{1}\right)$ and the dependence of the Poincaré lemma on parameters that

$$
\eta^{0}=\sum_{\nu=1}^{l} f_{\nu}(x, y) \sigma_{\nu}+d_{0} \alpha^{0}
$$

where $\alpha^{0}$ is a $(q-1)$-form on $M$, depending on $z$ and flat along $Y$, and where $f_{\nu}$ is flat along $y=0$ for $\nu=1, \cdots, l$. From (3.7) and (3.62), we have

$$
d_{0}\left(\eta^{1}+(-1)^{q} \alpha \frac{0}{z}\right)+\sum_{\nu} \frac{\partial f_{\nu}}{\partial \bar{z}}(z) \sigma_{\nu}=0 .
$$

Since the $\sigma_{\nu}$ 's generate $H^{q}(M)$, then (3.8) holds only when

$$
\frac{\partial f_{\nu}}{\partial \bar{z}}(z)=0, \quad \mu=1, \cdots, l .
$$

Since the holomorphic functions $f_{\nu}$ vanish along the $x$-axis, then

$$
f_{\nu}=0, \quad \nu=1, \cdots, l
$$

Let $\theta_{1}, \cdots, \theta_{k} \in \Omega^{q-1}\left(C^{\infty}(M)\right)$ be generators of $H^{q-1}(M)$. From (3.8) and (3.9) it follows that

$$
\eta^{1}+(-1)^{q} \alpha_{\bar{z}}^{0}=\sum_{\nu=1}^{k} g_{\nu}(z) \theta_{\nu}+d_{0} \beta^{1},
$$

where $\beta^{1}$ is a $(q-2)$-form on $M$ depending on $z$ and flat along $Y$, and where $g_{\nu} \in \mathcal{S}$ for all $\nu$. We have then

$$
\eta^{0}=d_{0} \alpha^{0} \quad \text { and } \quad \eta^{1}=(-1)^{q-1} \alpha_{z}^{0}+\sum_{\nu} g_{\nu}(z) \theta_{\nu}+d_{0} \beta^{1} .
$$

After substituting these expressions into (3.3) we obtain

$$
\eta=d\left(\alpha^{0}+\beta^{1} \wedge d \bar{z}\right)+\sum_{\nu} g_{\nu}(z) \theta_{\nu} \wedge d \bar{z} \bmod (d z)
$$

Hence $\eta$ is $d z$-cohomologous to the form

$$
\sum_{\nu=1}^{k} g_{\nu}(z) \theta_{\nu} \wedge d \bar{z}
$$

For every $\nu=1, \cdots, k$, let

$$
G_{\nu}(z)=\frac{1}{2 \pi i} \int_{\mathbb{C}} \frac{g_{\nu}(\mu)}{z-\mu} d \mu \wedge d \bar{\mu}
$$


and $H_{\nu}(z)$ be any germ of a $C^{\infty}$ function, holomorphic in the upper half plane. Then

$$
d\left(\sum_{\nu=1}^{k}(-1)^{q-1}\left(G_{\nu}(z)+H_{\nu}(z)\right) \theta_{\nu}\right)=\sum_{\nu=1}^{k} g_{\nu}(z) \theta_{\nu} \wedge d \bar{z} \quad \bmod (d z) .
$$

As in the proof of Lemma 1.1, the function $G_{\nu}+H_{\nu}$ is flat along the $x$-axis if and only if $g_{\nu}=0$. This shows that

$$
\sum_{\nu} g_{\nu}(z) \theta_{\nu} \wedge d \bar{z} \in B_{d z}^{q}(\mathcal{F}(Y)) \Longrightarrow\left(g_{\nu}=0, \quad \forall \nu\right) .
$$

The map $\Phi$ defined on $Z^{q} d z(\mathcal{F}(Y))$ by

$$
\Phi(\eta)=\sum_{\nu=1}^{k} g_{\nu}(z) \theta_{\nu}
$$

descends to an isomorphism from $H_{d z}^{q}(\mathcal{F}(Y))$ into $H^{q-1}(M) \otimes \mathcal{S}$. The proof of the proposition is complete.

\section{Proof of Proposition 1.2}

Let $\eta$ and $u$ be as in (1.10) and (1.11). Using the notation defined at the begining of section 3 , we can write

$$
u=u^{0}+u^{1} \wedge d \bar{z} \bmod (d z)
$$

and

$$
d u=d_{0} u^{0}+\left(d_{0} u^{1}+(-1)^{q-1} u_{\bar{z}}^{0}\right) \wedge d \bar{z} \bmod (d z) .
$$

This together with (1.10), (1.11) gives

$$
\begin{aligned}
d_{0} u^{0} & =0, \\
d_{0} u^{1}+(-1)^{q-1} u_{z}^{0} & =\sum_{\nu=1}^{k} f_{\nu}(z) \theta_{\nu} .
\end{aligned}
$$

From $\left(4.3_{1}\right)$ it follows that

$$
u^{0}=\sum_{\nu=1}^{k} a_{\nu}(z) \theta_{\nu}+d_{0} v^{0}
$$

where the $a_{\nu}$ are supported in $\overline{R^{+}}(\epsilon, \delta)$, and where $v^{0}$ is a $(q-2)$-form on $M$ depending on $z$ and flat along $Y$. With this $\left(4.3_{2}\right)$ becomes

$$
d_{0}\left(u^{1}+(-1)^{q-1} v_{\bar{z}}^{0}\right)+(-1)^{q-1} \sum_{\nu=1}^{k} \frac{\partial a_{\nu}}{\partial \bar{z}}(z) \theta_{\nu}=\sum_{\nu=1}^{k} f_{\nu}(z) \theta_{\nu} .
$$

Therefore

$$
\frac{\partial a_{\nu}}{\partial \bar{z}}(z)=(-1)^{q-1} f_{\nu}(z), \quad \nu=1, \cdots, k .
$$

and so

$$
a_{\nu}(z)=\frac{(-1)^{q-1}}{2 \pi i} \int_{\mathbb{C}} \frac{f_{\nu}(\mu)}{z-\mu} d \mu \wedge d \bar{\mu}+H_{\nu}(z),
$$

where $H_{\nu}(z)$ is holomorphic in the upper half plane. 
Now from (4.1) and (4.4), we can write

$$
u=\sum_{\nu=1}^{k} a_{\nu}(z) \theta_{\nu}+d_{0} v^{0} \bmod (d z, d \bar{z})
$$

from which (together with (1.11)) we obtain

$$
j_{x_{0}}^{*} u=\sum_{\nu=1}^{k} a_{\nu}\left(x_{0}, 0\right) \theta_{\nu}+d_{0}\left(j_{x_{0}}^{*} v^{0}\right)=0
$$

Therefore

$$
a_{\nu}\left(x_{0}, 0\right)=0 \quad \forall x_{0}, \quad \nu=1, \cdots, k .
$$

An argument similar to that in the proof of Lemma 1.1 shows that $a_{\nu}=0$ and consequently $f_{\nu}=0$. This proves the proposition.

\section{Proof of Proposition 1.3}

Consider the new variable $\sigma=s+p(t)$. Hence, $F=s+i \sigma$ and $\mathcal{E}_{x}[t, s]=\mathcal{E}_{x}[t, \sigma]$. For $\eta \in \Omega^{q}\left(\mathcal{E}_{x}[t, \sigma]\right), q \geq 1$, we write

$$
\eta=\eta^{0}+\eta^{1} \wedge d \bar{F} \bmod (d F),
$$

where $\eta^{0}$ and $\eta^{1}$ are $q$ - and $(q-1)$-forms in $t$-space with coefficients in $\mathcal{E}_{x}[t, \sigma]$. Hence,

$$
d \eta=d_{0} \eta^{0}+\left(d_{0} \eta^{1}+(-1)^{q} \eta_{\bar{F}}^{0}\right) \wedge d \bar{F} \bmod (d F),
$$

where $d_{0}$ is the exterior derivative in $t$-space and $\eta_{F}^{0}$ is the $q$ form obtained by differentiating the coefficients with respect to $\bar{F}$. If $\eta \in Z^{q} d F\left(\mathcal{E}_{x}[t, \sigma]\right)$, then

$$
\begin{aligned}
d_{0} \eta^{0} & =0, \\
d_{0} \eta^{1}+(-1)^{q} \eta_{F}^{0} & =0 .
\end{aligned}
$$

So

$$
\eta^{0}=d_{0} \alpha^{0}
$$

where $\alpha^{0}$ is a $(q-1)$-form in $t$-space with coefficients $\mathcal{E}_{x}([t, \sigma])$. And then,

$$
d_{0}\left(\eta^{1}+(-1)^{q} \alpha \frac{0}{F}\right)=0 .
$$

Now, if $q>1$, then

$$
\eta^{1}+(-1)^{q} \alpha_{\bar{F}}^{0}=d \beta^{1},
$$

where $\beta^{1}$ is a $(q-2)$-form in $t$-space with coefficients in $\mathcal{E}_{x}([x, \sigma])$. Therefore,

$$
\eta=d\left(\alpha^{0}+\beta^{1} \wedge d \bar{F}\right) \quad \bmod (d F),
$$

and the proposition is proved in this case. If $q=1$, then it follows from (5.5) that

$$
\eta^{1}-\alpha \frac{0}{F}=\sum_{j \geq 0} b_{j}(x) \sigma^{j}, \quad b_{j} \in \mathcal{E}_{x} .
$$

Let

$$
c_{j}(x)=\int_{0}^{x} b_{j}(s) d s
$$


It follows from (5.1), (5.4), (5.8), and (5.9) that

$$
\eta=d\left(\alpha^{0}+\sum_{j \geq 0} c_{j}(x) \sigma^{j}\right) \quad \bmod (d F)
$$

and the proposition is again proved here.

When $q=0$, it is easily verified that if

$$
\eta=\sum_{J \in \mathbb{Z}^{+n}, j \geq 0} f_{J, j}(x) t^{J} \sigma^{j}, \quad f_{J, j} \in \mathcal{E}_{x},
$$

is a first integral of $d F$, then

$$
f_{J, j}(x)=0, \quad \text { if } \quad J \neq 0 .
$$

Hence,

$$
\eta=\sum_{j \geq 0} a_{j}(x) \sigma^{j}, \quad a_{j} \in \mathcal{E}_{x} .
$$

Now from $d \eta \wedge d F=0$, we obtain

$$
a_{j+1}=i \frac{1}{j+1} \frac{d a_{j}}{d x}(x) \quad \forall j \geq 0 .
$$

That is,

$$
\eta=\sum_{j \geq 0} \frac{d^{j} a_{0}}{d x^{j}}(x) \frac{(i \sigma)^{j}}{j !} .
$$

The correspondence which to $\eta$ associates $a_{0}$ realizes the isomorphism between $H_{d F}^{0}\left(\mathcal{E}_{x}[t, s]\right)$ and $\mathcal{E}_{x}$. This completes the proof of the proposition.

\section{Mizohata structures in Dimension 2}

We recall Mizohata structures at $0 \in \mathbb{R}^{2}$ and the classifying space $\mathcal{D}_{0}^{+}$. For more details we refer to [Tr4]. A Mizohata structure at $0 \in \mathbb{R}^{2}$ can be thought of as the structure defined by a form

$$
\omega_{\rho}=d x+i(1+\rho(x, t)) d t^{2},
$$

where $\rho(x, t)$ is a $C^{\infty}$ function with support in $\{t \geq 0\}$. Notice that the function

$$
z^{-}(x, t)=x+i t^{2}
$$

satisfies

$$
d z^{-} \wedge \omega_{\rho}=0 \quad \text { in } \quad\{t \leq 0\} .
$$

Now we prove the following lemma

Lemma 6.1. There exists a $C^{\infty}$ function $z^{+}$defined in a rectangle

$$
R(\epsilon, \delta)=\left\{(x, t) \in \mathbb{R}^{2} ;|x| \leq \epsilon,|t| \leq \delta\right\}
$$

and satisfying the following conditions

(1) $d z^{+} \wedge \omega_{\rho}=0$ in $R^{+}(\epsilon, \delta)$, where $R^{ \pm}(\epsilon, \delta)=R(\epsilon, \delta) \cap\{t \geq 0$ (resp. $\left.t \leq 0)\right\}$;

(2) $d z^{+} \neq 0$ in $R(\epsilon, \delta)$;

(3) $z^{+}(x, 0) \in \mathbb{R}$, for $|x| \leq \epsilon$; and

(4) $z^{+}(-\epsilon, 0)=-\epsilon, z^{+}(0,0)=0, z^{+}(\epsilon, 0)=\epsilon$. 
Proof. Consider the smooth form $\alpha$, defined in the $(x, s)$-plane by

$$
\alpha(x, s)=d x+i(1+\rho(x, \sqrt{|s|})) d s .
$$

Since $\alpha$ is elliptic at 0 , then there are $C^{\infty}$ functions $f(x, s)$ and $g(x, s)$ defined near 0 such that

$$
\alpha(x, s)=g(x, s) d f(x, s) .
$$

Consider the map

$$
j: \mathbb{R}^{2} \longrightarrow \mathbb{R} \times \mathbb{R}^{+} ; \quad j(x, t)=\left(x, t^{2}\right) .
$$

Then,

$$
j^{*} \alpha=\omega_{\rho} \quad \text { in } \quad\{t \geq 0\} .
$$

Thus, the function

$$
F(x, t)=f\left(x, t^{2}\right)
$$

is a first integral of $\omega_{\rho}$ near 0 in $\{t \geq 0\}$.

For $\epsilon, \delta$ small positive numbers, let

$$
O(\epsilon, \delta)=F\left(R^{+}(\epsilon, \delta)\right) \quad \text { and } \quad S=F([-\epsilon, \epsilon] \times\{0\}) .
$$

Notice that $S$ is a part of the boundary of $O(\epsilon, \delta)$. By the Riemann mapping theorem, we can find a conformal mapping

$$
h: O(\epsilon, \delta) \longrightarrow h(O(\epsilon, \delta)) \subset\{\Im z \geq 0\}
$$

such that:

$$
h(S)=[-\epsilon, \epsilon], \quad h(F( \pm \epsilon, 0))= \pm \epsilon, \quad \text { and } \quad h(F(0,0))=0 .
$$

The function $z^{+}=h \circ F$ satisfies condition (1)-(4) of the lemma.

Let $C^{\infty}$ Dif $f_{0}^{+}$be the group of germs at $0 \in \mathbb{R}$ of increasing $C^{\infty}$ diffeomorphisms of $\mathbb{R}$ that preserve 0 and let $C^{\varpi}$ Dif $f_{0}^{+}$be the analogue in the real analytic category. Two elements $f, g \in C^{\infty}$ Dif $f_{0}^{+}$are said to be equivalent if there exist $h, k \in$ $C^{\varpi}$ Diff $f_{0}^{+}$such that

$$
g=h \circ f \circ k .
$$

The quotient space $\mathcal{D}_{0}^{+}$(space of Sjöstrand invariants) serves as a classifying space for the space $\operatorname{Miz}(2)$ of germs of Mizohata structures. The equivalence class in $\mathcal{D}_{0}^{+}$ of the germ of the function

$$
l(u)=z^{+} \circ\left(z^{-}\right)^{-1}(u)=z^{+}(u), \quad u \in \mathbb{R},
$$

represents uniquely the Mizohata structure defined by $\omega_{\rho}$.

\section{Mizohata StruCtures In High Dimensions}

After recalling the classification of germ of Mizohata structures by spaces $\mathcal{D}_{0}^{+}$ and $\mathcal{E}_{0}^{+}$as done in [Me2], we will compute the 0-th cohomology group, and close the section by the characterisation of the leaves of a Mizohata structure.

A germ of a Mizohata structure at $0 \in \mathbb{R}^{n+1}$ can be thought of as the germ of the structure given by by a form

$$
\omega=d x+i d q(t)+\sum_{j=1}^{n} a_{j}(x, t) d t_{j},
$$


where

$$
q(t)=t_{1}^{2}+\cdots+t_{k}^{2}-t_{k+1}^{2}-\cdots-t_{n}^{2}, \quad a_{j} \in \mathcal{F}_{0}(\{q(t)=0\}),
$$

and such that

$$
d \omega \wedge \omega=0 .
$$

The signature of this Mizohata structure is the signature of the quadratic form $q(t)$, that is, $\nu=|n-2 k|$. It is proved in [Me2] that if $\nu \neq|n-2|$, then the structure is locally integrable and then $\omega$ can be written, in a suitable system of coordinates, as

$$
\omega=d(x+i q(t))
$$

Otherwise the space $\mathcal{D}_{0}^{+}$(defined in section 6) and a space $\mathcal{E}_{0}^{+}$(to be described below) can be used to classify the space $M i z_{|n-2|}(n+1)$ of germs at $0 \in \mathbb{R}^{n+1}$ of Mizohata structures with signature $|n-2|$, when $n>2$ and $n=2$, respectively.

To explain this we start by considering the variety

$$
V_{0}=\left\{t \in \mathbb{R}^{n} ; q(t)=0\right\}
$$

(which reduces to the $x$-axis in the strictly pseudoconvex case, i.e. when the signature is $n$ ), and the connected components of $\mathbb{R}^{n+1} \backslash \mathbb{R} \times V_{0}$. If $\nu \neq|n-2|$, then

$$
\mathbb{R}^{n+1} \backslash \mathbb{R} \times V_{0}=\mathbb{R}_{+}^{n+1} \cup \mathbb{R}_{-}^{n+1},
$$

where

$$
\mathbb{R}_{+}^{n+1}=\{(x, t) ; q(t)>0\} \quad \text { and } \quad \mathbb{R}_{-}^{n+1}=\{(x, t) ; q(t)<0\} .
$$

If $\nu=n-2$ and $n>2$, then

$$
\mathbb{R}^{n+1} \backslash \mathbb{R} \times V_{0}=\mathbb{R}_{+,+}^{n+1} \cup \mathbb{R}_{+,-}^{n+1} \cup \mathbb{R}_{-}^{n+1},
$$

where

$$
\begin{gathered}
\mathbb{R}_{+,+}^{n+1}=\left\{q(t)>0, t_{1}>0\right\}, \quad \mathbb{R}_{+,-}^{n+1}=\left\{q(t)>0, t_{1}<0\right\}, \quad \text { and } \\
\mathbb{R}_{-}^{n+1}=\{q(t)<0\} .
\end{gathered}
$$

If $\nu=0$ and $n=2$, then

$$
\mathbb{R}^{n+1} \backslash \mathbb{R} \times V_{0}=\mathbb{R}_{+,+}^{3} \cup \mathbb{R}_{+,-}^{3} \cup \mathbb{R}_{-,+}^{3} \cup \mathbb{R}_{-,-}^{3},
$$

where

$$
\begin{array}{ll}
\mathbb{R}_{+,+}^{3}=\left\{t_{1}^{2}-t_{2}^{2}>0, t_{1}>0\right\}, & \mathbb{R}_{+,-}^{3}=\left\{t_{1}^{2}-t_{2}^{2}>0, t_{1}<0\right\} \\
\mathbb{R}_{-,+}^{3}=\left\{t_{1}^{2}-t_{2}^{2}<0, t_{2}>0\right\}, & \mathbb{R}_{-,-}^{3}=\left\{t_{1}^{2}-t_{2}^{2}<0, t_{2}<0\right\}
\end{array}
$$

Case $\nu=n-2$ and $n>2$. There exist coordinates $\left(x, t_{1}, \cdots, t_{n}\right)$ and a function $\rho(x, s)$ defined in the $(x, s)$-plane with support in $s \geq 0$ such that

$$
\begin{cases}\omega=\omega_{\rho}=d x+i(1+\rho(x, \sqrt{q(t)})) d q(t), & \text { in } \mathbb{R}_{+,+}^{n+1}, \\ \omega=\omega_{\rho}=d x+i d q(t), & \text { in } \mathbb{R}_{+,-}^{n+1} \cup \mathbb{R}_{-}^{n+1} .\end{cases}
$$

The two-dimensional Mizohata structure defined in the $(x, s)$-plane by

$$
\omega_{\rho}^{0}=d x+i(1+\rho(x, s)) d s^{2}
$$

determines $\omega_{\rho}$ in $\mathbb{R}^{n+1}$. Let $z^{ \pm}$be (semi) first integrals (in half planes) of $\omega_{\rho}^{0}$ as defined in section 6 and let $l=z^{+} \circ\left(z^{-}\right)^{-1}$. The class of $l$ in $\mathcal{D}_{0}^{+}$is the representative of $\omega_{\rho}^{0}$ and also that of $\omega_{\rho}$. 
Remark 7.1. The roles of $\mathbb{R}_{+,+}^{n+1}$ and $\mathbb{R}_{+,-}^{n+1}$ can be switched in (7.11); i.e., one can find a first integral of $\omega$ in $\mathbb{R}_{+,+}^{n+1} \cup \mathbb{R}_{-}^{n+1}$.

Case $\nu=0$ and $n=2$. In this situation, we can find coordinates $\left(x, t_{1}, t_{2}\right)$ and functions $\rho_{1}, \rho_{2}$ defined in the $(x, s)$-plane with support in $s \geq 0$ such that

$$
\begin{cases}\omega=\omega_{\rho_{1}, \rho_{2}}=d x+i\left(1+\rho_{1}\left(x, \sqrt{t_{1}^{2}-t_{2}^{2}}\right)\right) d\left(t_{1}^{2}-t_{2}^{2}\right), & \text { in } \mathbb{R}_{+,+}^{3} \\ \omega=\omega_{\rho_{1}, \rho_{2}}=d x+i\left(1+\rho_{2}\left(x, \sqrt{t_{2}^{2}-t_{1}^{2}}\right)\right) d\left(t_{1}^{2}-t_{2}^{2}\right), & \text { in } \mathbb{R}_{-,+}^{3} \\ \omega=\omega_{\rho_{1}, \rho_{2}}=d\left(x+i\left(t_{1}^{2}-t_{2}^{2}\right)\right), & \text { in } \mathbb{R}_{+,-}^{3} \cup \mathbb{R}_{-,-}^{3}\end{cases}
$$

The form $\omega=\omega_{\rho_{1}, \rho_{2}}$ in $\mathbb{R}^{3}$ is then determined by the two two-dimensional Mizohata structures $\omega_{\rho_{1}}^{0}$ and $\omega_{\rho_{2}}^{0}$ given in the $(x, s)$-plane by

$$
\begin{gathered}
\omega_{\rho_{1}}^{0}=d x+i\left(1+\rho_{1}(x, s)\right) d s^{2}, \\
\omega_{\rho_{2}}^{0}=d x-i\left(1+\rho_{2}(x, s)\right) d s^{2} .
\end{gathered}
$$

For $j=1,2$, let $z_{j}^{ \pm}$be the integrals of $\omega_{\rho_{j}}^{0}$ as defined in section 6 and let $l_{j}=$ $z_{j}^{+} \circ\left(z_{j}^{-}\right)^{-1}$.

Remark 7.2. One can find a first integral for $\omega$ in the union of a component of $q(t)>0$ and a component of $q(t)<0$. Hence, in the expression (7.13), the role of $\mathbb{R}_{ \pm,+}^{3}$ can be switched with that of $\mathbb{R}_{ \pm,-}^{3}$.

Now we proceed to define the classifying space $\mathcal{E}_{0}^{+}$. In $C^{\infty}$ Dif $f_{0}^{+} \times C^{\infty}$ Diff $f_{0}^{+}$, we define an equivalence relation by:

$$
(f, g) \sim\left(f^{\prime}, g^{\prime}\right) \Longleftrightarrow\left\{\begin{array}{c}
\exists a, b, c \in C^{\varpi} D i f f_{0}^{+} ; \\
f^{\prime}=a \circ f \circ c \text { and } g^{\prime}=b \circ g \circ c .
\end{array}\right.
$$

It is proved in [Me2] that the quotient, $\mathcal{E}_{0}^{+}$, thus obtained is in one to one correspondence with the space of Mizohata structures at $0 \in \mathbb{R}^{3}$ with signature 0 . A representative for $\omega_{\rho_{1}, \rho_{2}}$, as in (7.13), is given by the class of $\left(l_{1}, l_{2}\right)$ in $\mathcal{E}_{0}^{+}$.

We summarize this discussion as follows. A germ of a Mizohata structure at $0 \in \mathbb{R}^{n+1}$ with signature $\nu$ is given by (7.3) if $\nu \neq|n-2|$; by (7.11) if $\nu=n-2$ and $n>2$; and by (7.13) if $\nu=0$ and $n=2$.

For the description of $H_{\omega}^{0}$, we need to recall the principle of constancy along the fibers (see [BT] or [Tr4]). If $z$ is a first integral of a 1 -form $\omega$ in a neighborhood $O$ of a point $p$ with $d z(p) \neq 0$, then for any other $C^{\infty}$ first integral $f$ in $O$ there exist an open set $U \ni p, U \subset O$, and a $C^{\infty}$ function $h$ defined in $z(U)$, holomorphic in the interior of $z(U)$, such that

$$
f=h \circ z \quad \text { in } \quad U .
$$

From this principle, we can deduce the following for a Mizohata structure:

(1) $\nu \neq|n-2|$. Then

$$
H_{\omega}^{0}\left(\mathcal{E}^{n+1}\right) \cong \begin{cases}\mathbb{C}\{t\}, & \text { if } \nu \neq n \\ \mathcal{A}_{+}, & \text {if } \nu=n\end{cases}
$$


(2) $\nu=|n-2|$ and $n \neq 2$. Let $[l] \in \mathcal{D}_{0}^{+}$be a representative of $\omega$.

$$
\begin{aligned}
& \text { If }[l]=[\text { identity }], \text { then } \begin{cases}H_{\omega}^{0}\left(\mathcal{E}^{n+1}\right) \cong \mathbb{C}\{z\}, & \text { if } n>2, \\
H_{\omega}^{0}\left(\mathcal{E}^{1}\right) \cong \mathcal{A}_{+}, & \text {if } n=1 ;\end{cases} \\
& \text { if }[l] \neq \text { identity], then } H_{\omega}^{0}\left(\mathcal{E}^{n+1}\right) \cong \mathbb{C} .
\end{aligned}
$$

(3) $\nu=0$ and $n=2$. Let $\left[l_{1}, l_{2}\right] \in \mathcal{E}_{0}^{+}$be a representative of $\omega$.

$$
\begin{aligned}
& \text { If }\left[l_{1}, l_{2}\right]=[\mathrm{id}, \mathrm{id}], \text { then } H_{\omega}^{0}\left(\mathcal{E}^{3}\right) \cong \mathbb{C}\{z\} ; \\
& \text { if }\left[l_{1}, l_{2}\right] \neq[\mathrm{id}, \mathrm{id}] \text {, then } H_{\omega}^{0}\left(\mathcal{E}^{3}\right) \cong \mathbb{C} \text {. }
\end{aligned}
$$

We close this section with the topological description of $V_{0}$ (given by (7.4)) and of the leaves of $\omega$. Let

$$
V^{+}=\left\{t \in \mathbb{R}^{n} ; q(t)>0\right\} \quad \text { and } \quad V^{-}=\left\{t \in \mathbb{R}^{n} ; q(t)<0\right\} .
$$

Define $\Sigma^{0}, \Sigma^{+}$, and $\Sigma^{-}$as

$$
\Sigma^{0}=V_{0} \cap S^{n-1}, \quad \text { and } \quad \Sigma^{ \pm}=V^{ \pm} \cap S^{n-1},
$$

where $S^{n-1}=\left\{t \in \mathbb{R}^{n} ;|t|=1\right\}$ is the $(n-1)$ sphere. Denote by $B^{m}$ the unit ball in $\mathbb{R}^{m}$.

Lemma 7.1. Assume that $\nu \neq n$. Then we have the following isomorphisms:

$$
\Sigma^{0} \cong S^{k-1} \times S^{n-k-1}, \quad \Sigma^{+} \cong S^{k-1} \times B^{n-k}, \quad \text { and } \quad \Sigma^{-} \cong B^{k} \times S^{n-k-1} .
$$

Proof. A point $\theta=\left(\theta_{1}, \cdots, \theta_{n}\right) \in S^{n-1}$ will be written as $\theta=\left(\theta^{\prime}, \theta^{\prime \prime}\right)$, where $\theta^{\prime}=$ $\left(\theta_{1}, \cdots, \theta_{k}\right)$ and $\theta^{\prime \prime}=\left(\theta_{k+1}, \cdots, \theta_{n}\right)$. It is then easily verified that the following are diffeomorphisms:

$$
\begin{aligned}
\phi^{0}: S^{k-1} \times S^{n-k-1} \longrightarrow \Sigma^{0} ; & \phi^{0}(\alpha, \beta) & =\frac{1}{\sqrt{2}}(\alpha, \beta), \\
\phi^{+}: S^{k-1} \times B^{n-k} \longrightarrow \Sigma^{+} ; & \phi^{+}(\alpha, \tau) & =\frac{1}{\sqrt{2}}\left(\sqrt{2-|\tau|^{2}} \alpha, \tau\right), \\
\phi^{-}: B^{k} \times S^{n-k-1} \longrightarrow \Sigma^{-} ; & \phi^{-}(\sigma, \beta) & =\frac{1}{\sqrt{2}}\left(\sigma, \sqrt{1-|\sigma|^{2}} \beta\right) .
\end{aligned}
$$

\section{Reduction to Flatness AlOng the $x$-AXis}

We prove here that an $\omega$-closed form is $\omega$-cohomologous to a form flat along the $x$-axis. More precisely,

Proposition 8.1. Suppose that $\omega$ defines a Mizohata structure. Then for every $\eta \in Z^{q}{ }_{\omega}\left(\mathcal{E}^{n+1}\right)$ there exists $u \in \Omega^{q-1}\left(\mathcal{E}^{n+1}\right)$ such that

$$
\eta-d u \in Z^{q}{ }_{\omega}\left(\mathcal{F}_{0}(\{t=0\})\right) .
$$

Proof. First assume that $\omega$ is locally integrable, i.e.,

$$
\omega=d(x+i q(t)) .
$$

Then from

$$
d \eta \wedge \omega=0
$$

it follows that

$$
d \eta=\eta_{1} \wedge \omega
$$


for some $\eta_{1} \in \Omega^{q}\left(\mathcal{E}^{n+1}\right)$. By taking the exterior derivative we see that $\eta_{1} \in$ $Z^{q}{ }_{\omega}\left(\mathcal{E}^{n+1}\right)$, and so there exists $\eta_{2} \in \Omega^{q}\left(\mathcal{E}^{n+1}\right)$ such that

$$
d \eta_{1}=\eta_{2} \wedge \omega
$$

The continuation of this process generates a sequence $\left\{\eta_{j}\right\}_{j \geq 0} \subset \Omega^{q}\left(\mathcal{E}^{n+1}\right)$ (with $\left.\eta_{0}=\eta\right)$ such that

$$
d \eta_{j}=\eta_{j+1} \wedge \omega
$$

(this is known as a Godbillon-Vey sequence associated with $\omega$ ). Now we define a formal $q$-form $\alpha$ in the $(x, t, s)$-space (where $s$ is real) by

$$
\alpha=\sum_{j=0}^{\infty} \eta_{j} \frac{(i s)^{j}}{j !}
$$

From (8.1), we easily derive

$$
d \alpha=\left(\sum_{j \geq 0} \eta_{j+1} \frac{(i s)^{j}}{j !}\right) \wedge d(x+i(q(t)+s)) .
$$

Thus,

$$
d \alpha \wedge d(x+i(q(t)+s))=0
$$

Consider the formal expansion of the $\eta_{j}$ 's with respect to $t$, and the formal expansion of $\alpha$ with respect to $t$ and $s$, i.e.

$$
\begin{aligned}
T_{t} \eta_{j} & =\sum_{r \in\left(\mathbb{Z}^{+}\right)^{n}} \eta_{j}^{r} t^{r}, \\
T_{t, s} \alpha & =\sum_{j \geq 0, r \in\left(Z^{+}\right)^{n}} \eta_{j}^{r} \frac{t^{r}(i s)^{j}}{j !} .
\end{aligned}
$$

( $\eta_{j}^{r}$ is a $q$-form with coefficients depending only on $x$.) It follows from (8.4) that

$$
T_{t, s} \alpha \in Z_{d(x+i(q(t)+s))}^{q}\left(\mathcal{E}_{x}[t, s]\right) .
$$

From Proposition 1.3, we can find

$$
\hat{\beta} \in \Omega^{q-1}\left(\mathcal{E}_{x}[t, s]\right)
$$

such that

$$
\left(T_{t, s} \alpha-d \hat{\beta}\right) \wedge d(x+i(q(t)+s))=0 .
$$

By Borel's extension theorem (see [GG] for example), we can find $\beta \in \Omega^{q-1}\left(\mathcal{E}^{n+2}\right)$ such that

$$
T_{t, s} \beta=\hat{\beta} .
$$

Substituting this into (8.6), we get

$$
T_{t, s}(\alpha-d \beta) \wedge d(x+i(q(t)+s))=0 .
$$

Let $\beta_{0}$ be the pullback of $\beta$ to the subspace $s=0$. Since the pullback of $\alpha$ is $\eta_{0}=\eta$, then it follows from (8.8) that

$$
T_{t}\left(\eta-d \beta_{0}\right) \wedge d(x+i q(t))=0,
$$

which means $\eta-d \beta_{0} \in Z^{q}{ }_{\omega}\left(\mathcal{F}_{0}(\{t=0\})\right)$, and the proposition is proved in this case. 
When $\omega$ is not locally integrable (which might happen when $\nu=|n-2|)$ we know from section 7 that $\omega$ coincides with $d(x+i q(t))$ in components of $\mathbb{R}^{n+1} \backslash \mathbb{R} \times V_{0}$. The existence of the sequence $\eta_{j}$ is then guaranteed in those components and the rest of the proof can be continued verbatim.

\section{Reduction to flatness AlOng $\mathbb{R} \times V_{0}$}

We prove here that for a Mizohata structure given by $\omega$, the $\omega$-closed forms are $\omega$-cohomologous to forms flat along the separatrix. More precisely, we have the following

Proposition 9.1. Let $\eta \in Z^{q}{ }_{\omega}\left(\mathcal{E}^{n+1}\right)$ with $q \geq 1$. Then there exists $u \in \Omega^{q-1}\left(\mathcal{E}^{n+1}\right.$ such that

$$
\eta-d u \in Z^{q}{ }_{\omega}\left(\mathcal{F}_{0}(\{q(t)=0\})\right) .
$$

Since the case $\nu=n$ is contained in Proposition 8.1, we will assume that $\nu \neq n$. The idea of the proof is to use the desingularization of the variety $V^{0}$ to construct a manifold in which the pullback of $q(t)$ can be considered as a coordinate $y$. Then expansion with respect to $y$ together with Borel's extension theorem will allow the reduction to forms that are flat along $y=0$ and so, after pushforward, to forms that are flat along $q(t)=0$. The remainder of this section gives the details of this.

We start with the case $\nu \neq|n-2|$ (and so $\omega=d(x+i q)$ ). Let

$$
\Pi_{1}: \mathbb{R} \times \overline{\mathbb{R}^{+}} \times S^{n-1} \longrightarrow \mathbb{R} \times \mathbb{R}^{n} ; \quad \Pi_{1}(x, r, \theta)=(x, r \theta) .
$$

Note that if $\eta \in \Omega^{q}\left(\mathcal{E}^{n+1}\right)$, then its pullback

$$
\eta_{1}=\Pi_{1}^{*} \eta
$$

is the germ of a $q$-form along

$$
\{0\} \times\{0\} \times S^{n-1} \subset \mathbb{R} \times \overline{\mathbb{R}^{+}} \times S^{n-1} .
$$

Let $q_{0}$ be the restriction to the sphere $S^{n-1}$ of the quadratic form $q(t)$ and let

$$
\begin{aligned}
\Delta^{0} & =\overline{\Pi_{1}^{-1}\left(\mathbb{R} \times V_{0} \backslash \mathbb{R} \times\{0\}\right)} \cap \mathbb{R} \times\{0\} \times S^{n-1} \\
& =\left\{(x, r, \theta) \in \mathbb{R} \times \overline{\mathbb{R}^{+}} \times S^{n-1} ; r=0 \text { and } q_{0}(\theta)=0\right\} \quad \text { and } \\
\Delta^{ \pm} & =\overline{\Pi_{1}^{-1}\left(\mathbb{R} \times V^{ \pm} \backslash \mathbb{R} \times\{0\}\right)} \cap \mathbb{R} \times\{0\} \times S^{n-1} \\
& =\left\{(x, r, \theta) \in \mathbb{R} \times \overline{\mathbb{R}^{+}} \times S^{n-1} ; r=0 \text { and } q_{0}(\theta) \geq 0(\text { resp. } \leq 0)\right\} .
\end{aligned}
$$

Hence,

$$
\begin{aligned}
& \Delta^{0}=\mathbb{R} \times\{0\} \times \Sigma^{0} \cong \mathbb{R} \times S^{k-1} \times S^{n-k-1} \\
& \Delta^{+}=\mathbb{R} \times\{0\} \times \bar{\Sigma}^{+} \cong \mathbb{R} \times S^{k-1} \times \overline{B^{n-k}} \\
& \Delta^{-}=\mathbb{R} \times\{0\} \times \bar{\Sigma}^{-} \cong \mathbb{R} \times \overline{B^{k}} \times S^{n-k-1}
\end{aligned}
$$

Let $a>0$ be small enough so that the tubular neighborhood

$$
U_{a}=q_{0}^{-1}((-a, a))
$$

of $\Sigma^{0}$ in $S^{n-1}$ is diffeomorphic to $\Sigma^{0} \times(-a, a)$. Let

$$
\Psi_{1}: \Sigma^{0} \times(-a, a) \longrightarrow U_{a} ; \quad \Psi_{1}\left(\left(\theta^{\prime}, \theta^{\prime \prime}\right), s\right)=\left(\sqrt{s+1} \theta^{\prime}, \sqrt{1-s} \theta^{\prime \prime}\right) .
$$


Notice that

$$
q_{0} \circ \Psi_{1}\left(\left(\theta^{\prime}, \theta^{\prime \prime}\right), s\right)=s .
$$

Let

$$
\begin{gathered}
\Pi_{2}: \mathbb{R} \times \overline{\mathbb{R}^{+}} \times \Sigma^{0} \times(-a, a) \longrightarrow \mathbb{R} \times \overline{\mathbb{R}^{+}} \times U_{a} ; \\
\Pi_{2}\left(x, r,\left(\theta^{\prime}, \theta^{\prime \prime}\right), s\right)=\left(x, r, \Psi_{1}\left(\left(\theta^{\prime}, \theta^{\prime \prime}\right), s\right)\right) .
\end{gathered}
$$

Note that if $\eta_{1}$ is a form in $\mathbb{R} \times \overline{\mathbb{R}^{+}} \times U_{a}$, flat along $r=0$, then its pullback

$$
\eta_{2}=\Pi_{2}^{*} \eta_{1}
$$

defined in $\mathbb{R} \times \overline{\mathbb{R}^{+}} \times \Sigma^{0} \times(-a, a)$ is flat along $r=0$. Note also that

$$
\Pi_{2}^{*}\left(\Pi_{1}^{*} d(x+i q(t))\right)=d\left(x+i r^{2} s\right) .
$$

Let

$$
\begin{gathered}
\Psi_{2}: \overline{\mathbb{R}^{+}} \times \mathbb{R} \longrightarrow \overline{\mathbb{R}^{+}} \times \mathbb{R} ; \\
\Psi(r, s)=(u, y) \text { with } \begin{cases}u & =r, \\
y & =r^{2} s .\end{cases}
\end{gathered}
$$

Notice that $\Psi_{2}^{-1}(u, y)=\left(u, \frac{y}{u^{2}}\right)$ is a homeomorphism away from $u=0$.

Remark 9.1. If $f(r, s)$ is the germ at $0 \in \overline{\mathbb{R}^{+}} \times \mathbb{R}$ of a $C^{\infty}$ function, flat along $r=0$, then its pullback via $\Psi_{2}^{-1}$,

$$
\left(\Psi_{2}^{-1}\right)^{*} f(u, y)=f\left(u, \frac{y}{u^{2}}\right),
$$

can be extended as a $C^{\infty}$ function to a full neighborhood of 0 in the $(u, y)$-plane. Moreover this extension can be taken to be flat along the $y$-axis.

Let

$$
\begin{gathered}
\Pi_{3}: \mathbb{R} \times \overline{\mathbb{R}^{+}} \times[-a, a] \times \Sigma^{0} \longrightarrow \mathbb{R} \times \mathbb{R}^{2} \times \Sigma^{0}, \\
\Pi_{3}(x, r, s, \theta)=\left(x, \Psi_{2}(r, s), \theta\right) .
\end{gathered}
$$

For $\delta$ and $\epsilon$ small positive numbers, define

$$
\begin{aligned}
R(\delta, a) & =[0, \delta] \times[-a, a], \\
A(\delta, a, \epsilon) & =\Pi_{3}\left([-\epsilon, \epsilon] \times R(\delta, a) \times \Sigma^{0}\right) \\
& =[-\epsilon, \epsilon] \times \Psi_{2}(R(\delta, a)) \times \Sigma^{0} .
\end{aligned}
$$

It follows from Remark 9.1 that if $\eta_{2}$ is a $C^{\infty}$ form defined in a neighborhood of

$$
\{0\} \times\{0\} \times\{0\} \times \Sigma^{0} \subset \mathbb{R} \times \overline{\mathbb{R}^{+}} \times[-a, a] \times \Sigma^{0}
$$

and flat along $r=0$, then its pullback

$$
\eta_{3}=\left(\Pi_{3}^{-1}\right)^{*} \eta_{2},
$$

which is defined in a neighborhood of

$$
\{0\} \times\{0\} \times \Sigma^{0} \subset A(\delta, a, \epsilon),
$$

can be extended as a $C^{\infty}$ form to a full neighborhood of

$$
\{0\} \times\{0\} \times \Sigma^{0} \subset \mathbb{R} \times \mathbb{R}^{2} \times \Sigma^{0} .
$$


Moreover this extension $\widetilde{\eta}_{3}$ of $\eta_{3}$ can be taken to be flat along $u=0$. Notice that

$$
d z=\left(\Pi_{3}^{-1}\right)^{*}\left(\Pi_{2}^{*}\left(\Pi_{1}^{*} d(x+i q(t))\right)\right)=d(x+i y) .
$$

With all of this in mind, choose $\eta \in Z^{q}{ }_{\omega}\left(\mathcal{E}^{n+1}\right)$. From proposition 8.1, we can assume that $\eta \in Z^{q}{ }_{\omega}\left(\mathcal{F}_{0}(\{t=0\})\right)$. Then, $\eta_{1}=\Pi_{1}^{*} \eta$ is flat along $r=0, \eta_{2}=\Pi_{2}^{*} \eta_{1}$ is flat along $r=0$, and $\widetilde{\eta}_{3}=\left(\widetilde{\Pi_{3}^{-1}}\right)^{*} \eta_{2}$ is flat along $u=0$. Moreover, since $\eta$ is $\omega$-closed, then

$$
d \widetilde{\eta}_{3} \wedge d(x+i y)=0 \quad \text { in } \quad A(\delta, a, \epsilon) .
$$

Note that (9.14) may not hold in a full neighborhood of $\{0\} \times\{0\} \times \Sigma^{0}$ in $\mathbb{R} \times \mathbb{R}^{2} \times \Sigma^{0}$.

Let

$$
T_{y} \widetilde{\eta}_{3}=\sum_{j \geq 0} \eta_{3}^{j} y^{j}
$$

be the expansion of $\widetilde{\eta}_{3}$ with respect to the variable $y$. Each $\eta_{3}^{j}$ is then a $q$-form with coefficients independent on $y$ and flat along $u=0$. We have

$$
d\left(T_{y} \widetilde{\eta}_{3}\right)=\sum_{j \geq 0}\left\{d \eta_{3}^{j}+\frac{i}{2}(j+1) \eta_{3}^{j+1} \wedge d \bar{z}\right\} y^{j} \quad \bmod (d z) .
$$

We write

$$
\eta_{3}^{j}=A^{j}+B^{j} \wedge d \bar{z}+C^{j} \wedge d u+D^{j} \wedge d \bar{z} \wedge d u \bmod (d z),
$$

where $A^{j}, B^{j}, C^{j}$, and $D^{j}$ are forms on $\Sigma^{0}$ with respective degrees $q, q-1, q-1$, and $q-2$, depending on parameter $(x, u)$ and flat along $u=0$.

For a $q$-form $\alpha$ on $\Sigma^{0}$ depending on $(x, u)$ we adopt the following notation: $d_{0}$ will be the exterior derivative on $\Sigma^{0}, \alpha_{u}$ and $\alpha_{\bar{z}}$ will be the $q$-forms on $\Sigma^{0}$ obtained from $\alpha$ by differentiating the coefficients with respect to $u$ and $\bar{z}$, respectively. When $\alpha$ is considered as a $q$-form on $\mathbb{R} \times \mathbb{R}^{2} \times \Sigma^{0}$, then

$$
d \alpha=d_{0} \alpha+(-1)^{q} \alpha_{\bar{z}} \wedge d \bar{z}+(-1)^{q} \alpha_{u} \wedge d u \bmod (d z) .
$$

With this, we obtain from (9.17)

$$
\begin{aligned}
d \eta_{3}^{j}= & d_{0} A^{j}+\left(d_{0} B^{j}+(-1)^{q} A_{\bar{z}}^{j}\right) \wedge d \bar{z}+\left(d_{0} C^{j}+(-1)^{q} A_{u}^{j}\right) \wedge d u \\
& +\left(d_{0} D^{j}+(-1)^{q} B_{u}^{j}+(-1)^{q-1} C_{\bar{z}}^{j}\right) \wedge d \bar{z} \wedge d u \quad \bmod (d z) .
\end{aligned}
$$

Since,

$$
\eta_{3}^{j+1} \wedge d \bar{z}=A^{j+1} \wedge d \bar{z}-C^{j+1} \wedge d \bar{z} \wedge d u \bmod (d z)
$$

then

$$
\begin{gathered}
d \eta_{3}^{j}+\frac{i}{2}(-1)^{q}(j+1) \eta_{3}^{j+1} \wedge d \bar{z}=d_{0} A^{j}+\left(d_{0} C^{j}+(-1)^{q} A_{u}^{j}\right) \wedge d u \\
+\left(d_{0} B^{j}+(-1)^{q} A_{\bar{z}}^{j}+(-1)^{q} \frac{i}{2}(j+1) A^{j+1}\right) \wedge d \bar{z} \\
+\left(d_{0} D^{j}+(-1)^{q} B_{u}^{j}+(-1)^{q-1} C_{\bar{z}}^{j}-(-1)^{q} \frac{i}{2}(j+1) C^{j+1}\right) \wedge d \bar{z} \wedge d u \bmod (d z) .
\end{gathered}
$$


Now it follows from (9.14), (9.16) and (9.20) that

$$
\begin{aligned}
& d_{0} A^{j}=0, \\
& d_{0} B^{j}+(-1)^{q} A_{z}^{j}+(-1)^{q} \frac{i}{2}(j+1) A^{j+1}=0, \\
& d_{0} C^{j}+(-1)^{q} A_{u}^{j}=0, \\
& d_{0} D^{j}+(-1)^{q} B_{u}^{j}+(-1)^{q-1} C_{\bar{z}}^{j}-(-1)^{q} \frac{i}{2}(j+1) C^{j+1}=0 .
\end{aligned}
$$

Let $\pi_{1}, \pi_{2}, \cdots$ be generators of $H^{q}\left(\Sigma^{0}\right)$. From $\left(9.21_{1}\right)$ we get

$$
A^{j}=\sum_{l} a_{l}^{j}(x, u) \pi_{l}+d_{0} \alpha^{j}
$$

where $\alpha^{j}$ is a $(q-1)$-form on $\Sigma^{0}$, flat along $u=0$, and where the $a_{l}^{j}$,s are $C^{\infty}$ functions of $x, u$ and flat along $u=0$. With this, $\left(9.21_{3}\right)$ becomes

$$
d_{0}\left(C^{j}+(-1)^{q} \alpha_{u}^{j}\right)+(-1)^{q} \sum_{l} \frac{\partial a_{l}^{j}}{\partial u} \pi_{l}=0 .
$$

Hence,

$$
\frac{\partial a_{l}^{j}}{\partial u}=0 \quad \text { and } \quad d_{0}\left(C^{j}+(-1)^{q} \alpha_{u}^{j}\right)=0
$$

from which it follows at once that

$$
a_{l}^{j}(x, u)=0
$$

(since $\left.a_{l}^{j}(x, 0)=0\right)$ and that

$$
C^{j}+(-1)^{q} \alpha_{u}^{j}=\sum_{l} g_{l}^{j}(x, u) \delta_{l}+d_{0} \gamma^{j},
$$

where the $\delta_{l}$ 's are generators of $H^{q-1}\left(\Sigma^{0}\right), g_{l}^{j}(x, u)$ smooth functions flat along $u=0$, and $\gamma^{j}$ a $(q-2)$-form on $\Sigma^{0}$ flat along $u=0$. With (9.25) and (9.22), we can rewrite $\left(9.21_{2}\right)$ as

$$
d_{0}\left(B^{j}+(-1)^{q} \alpha_{\bar{z}}^{j}+(-1)^{q} \frac{i}{2}(j+1) \alpha^{j+1}\right)=0
$$

and then

$$
B^{j}+(-1)^{q} \alpha_{\bar{z}}^{j}+\frac{i}{2}(j+1) \alpha^{j+1}=\sum_{l} f_{l}^{j}(x, u) \delta_{l}+d_{0} \beta^{j},
$$

where $\beta^{j}$ is a $(q-2)$-form on $\Sigma^{0}$, flat along $u=0$, and where the $f_{l}^{j}$,s are smooth functions of $(x, u)$ and flat along $u=0$. With (9.22), (9.25), (9.26) and (9.27), the expression in $\left(9.21_{4}\right)$ becomes

$$
\begin{aligned}
d_{0}\left(D^{j}\right. & \left.+(-1)^{q} \beta_{u}^{j}+(-1)^{q-1} \gamma_{\bar{z}}^{j}-(-1)^{q} \frac{i}{2}(j+1) \gamma^{j+1}\right) \\
= & (-1)^{q-1} \sum_{l}\left(\frac{\partial f_{l}^{j}}{\partial u}-\frac{\partial g_{l}^{j}}{\partial \bar{z}}-\frac{i}{2}(j+1) g_{l}^{j+1}\right) \delta_{l} .
\end{aligned}
$$


Therefore,

$$
\begin{aligned}
\frac{\partial f_{l}^{j}}{\partial u}-\frac{\partial g_{l}^{j}}{\partial \bar{z}}-\frac{i}{2}(j+1) g_{l}^{j+1} & =0, \\
d_{0}\left(D^{j}+(-1)^{q} \beta_{u}^{j}+(-1)^{q-1} \gamma_{\bar{z}}^{j}-(-1)^{q} \frac{i}{2}(j+1) \gamma^{j+1}\right) & =0 .
\end{aligned}
$$

Thus,

$$
D^{j}+(-1)^{q}\left(\beta_{u}^{j}-\gamma_{\bar{z}}^{j}-\frac{i}{2}(j+1) \gamma^{j+1}\right)=\sum_{l} m_{l}^{j}(x, u) \tau_{l}+d \mu^{j},
$$

where the $\tau_{l}$ 's are generators of $H^{q-2}\left(\Sigma^{0}\right)$, the $m_{l}^{j}$ are smooth functions flat along $u=0$, and $\mu^{j}$ a $(q-3)$-form on $\Sigma^{0}$ flat along $u=0$. Let

$$
\sigma^{j}=\alpha^{j}+\beta^{j} \wedge d \bar{z}+\gamma^{j} \wedge d u+\sum_{l}\left(\int_{0}^{u} m_{l}^{j}(x, s) d s\right) \tau_{l}+\mu^{j} .
$$

Then, it follows from (9.32), (9.31), (9.27), (9.25), (9.22), and (9.17) that

$$
\begin{gathered}
\eta_{3}^{j}=d \sigma^{j}+(-1)^{q-1} \frac{i}{2}(j+1) \alpha^{j+1} \wedge d \bar{z}+(-1)^{q} \frac{i}{2}(j+1) \gamma^{j+1} \wedge d \bar{z} \wedge d u \\
+(-1)^{q-1} \sum_{l}\left(f_{l}^{j}(x, u) d \bar{z}+g_{l}^{j}(x, u) d u\right) \wedge \delta_{l} .
\end{gathered}
$$

For every $j$ and $l$, define a function $\rho_{l}^{j}(x, u)$ (flat along $u=0$ ) by

$$
\rho_{l}^{j}(x, u)=\int_{0}^{u} g_{l}^{j}(x, s) d s .
$$

It follows from (9.29) and (9.34) that

$$
d \rho_{l}^{j}=f_{l}^{j}(x, u) d \bar{z}+g_{l}^{j}(x, u) d u-\frac{i}{2}(j+1) \rho_{l}^{j+1}(x, u) d \bar{z} \bmod (d z) .
$$

Define the formal $(q-1)$-form $\hat{\phi}$, with coefficients flat along $u=0$, by

$$
\hat{\phi}=\sum_{j \geq 0}\left\{\sigma^{j}+\sum_{l} \rho_{l}^{j}(x, u) \delta_{l}\right\} y^{j} .
$$

It is verified at once that

$$
d \hat{\phi}=T_{y} \widetilde{\eta}_{3} \bmod (d z) .
$$

Let $\phi$ be a smooth $(q-1)$-form such that

$$
T_{y} \phi=\hat{\phi} \text {. }
$$

Notice that $\phi$ is necessarily flat along $u=0$ and that

$$
\left(\widetilde{\eta}_{3}-d \phi\right) \wedge d(x+i y) \text { is flat along } u y=0 .
$$

Now $\psi=\left(\Pi_{2}^{-1}\right)^{*} \Pi_{3}^{*} \phi$ is a $C^{\infty}$ form flat along $r=0$ in $\mathbb{R} \times \overline{\mathbb{R}^{+}} \times U_{a}$. We can extended it as a $C^{\infty}$ form $\widetilde{\psi}$ to a full neighborhood of

$$
\{0\} \times\{0\} \times S^{n-1} \subset \mathbb{R} \times \overline{\mathbb{R}^{+}} \times S^{n-1}
$$

in such a way that it is flat along $\Pi_{1}^{-1}(\mathbb{R} \times\{0\})$. Hence,

$$
f=\left(\Pi_{1}^{-1}\right)^{*} \widetilde{\psi}
$$


is a smooth $(q-1)$-form defined in a neighborhood of $0 \in \mathbb{R}^{n+1}$. Moreover, it follows from (9.39) that

$$
(\eta-d f) \wedge \omega \in \Omega^{q}\left(\mathcal{F}_{0}(\{q(t)=0\})\right) .
$$

The proposition is proved in this case.

The case $\nu=n-2, n>2$, can be handled in an analogous way with the following modifications. Each $\Delta^{0}$ and $U_{a}$ has now 2 components $\Delta_{+}^{0}, \Delta_{-}^{0}$ and $U_{a,+}, U_{a,-}$, respectively, and

$$
\Pi_{1}\left(\mathbb{R} \times \overline{\mathbb{R}^{+}} \times U_{a,+}\right) \subset\left\{t_{1} \geq 0\right\} \quad \text { and } \quad \Pi_{1}\left(\mathbb{R} \times \overline{\mathbb{R}^{+}} \times U_{a,-}\right) \subset\left\{t_{1} \leq 0\right\} .
$$

Using remark 7.1 we can assume

$$
\Pi_{1}^{*} \omega=d\left(x+i r^{2} q_{0}(\theta)\right) \quad \text { in } \mathbb{R} \times \overline{\mathbb{R}^{+}} \times U_{a,+} \text { or in } \mathbb{R} \times \overline{\mathbb{R}^{+}} \times U_{a,-} .
$$

The same argument as that in the previous case yields $(q-1)$-forms $\psi_{ \pm}$in $\mathbb{R} \times$ $\overline{\mathbb{R}^{+}} \times U_{a, \pm}$ flat along $r=0$, such that

$$
\left(\eta-d \psi_{ \pm}\right) \wedge \Pi_{1}^{*} \omega \quad \text { is flat along } \quad \mathbb{R} \times \overline{\mathbb{R}^{+}} \times \Delta_{ \pm}^{0} .
$$

Now the forms $\psi_{ \pm}$can be extended as forms $\widetilde{\psi}_{ \pm}$defined in a full neighborhood of

$$
\{0\} \times\{0\} \times S^{n-1} \subset \mathbb{R} \times \overline{\mathbb{R}^{+}} \times S^{n-1}
$$

and flat along $r=0$ and along $\mathbb{R} \times \overline{\mathbb{R}^{+}} \times \Delta_{\mp}^{0}$. If we define $f$ as

$$
f=\left(\Pi_{1}^{-1}\right)^{*}\left(\widetilde{\psi}_{+}+\widetilde{\psi}_{-}\right) \in \Omega^{q-1}\left(\mathcal{F}_{0}(\{t=0\})\right),
$$

then

$$
(\eta-d f) \wedge \omega \in \Omega^{q}\left(\mathcal{F}_{0}(\{q(t)=0\})\right) .
$$

The proposition is again proved in this situation.

The last case $\nu=0$ and $n=2$ can be dealt with in a similar way.

\section{Cohomology With Support in A WedGe}

We consider here the cohomology groups obtained when the forms have support in the components of $\mathbb{R}^{n+1} \backslash \mathbb{R} \times V_{0}$.

Let $\omega$ be a form defining a germ of a Mizohata structure and given by the canonical expression (7.11) or (7.13) of section 7 . Let $W_{1}, W_{2}, \cdots$ be the connected components of $\mathbb{R}^{n+1} \backslash \mathbb{R} \times V_{0}$. For each $i$, let $C_{0}\left(W_{i}\right)$ be the space of germs at $0 \in \mathbb{R}^{n+1}$ of $C^{\infty}$ functions with support in $\overline{W_{i}}$ and flat along $\mathbb{R} \times V_{0}$, i.e.

$$
C_{0}\left(W_{i}\right)=\left\{f \in \mathcal{E}^{n+1} ; f=0 \text { in } \mathbb{R}^{n+1} \backslash W_{i}\right\} \cap \mathcal{F}_{0}(\{q(t)=0\}) .
$$

We can assume (remarks 7.1 and 7.2) that

$$
\omega=d(x+i q(t)) \quad \text { in } W_{i} .
$$

Let $L_{i}$ be a (generic) leaf of $\omega$ in $W_{i}\left(L_{i}\right.$ is a level set of $\left.x+i q(t)\right)$. Note that when the signature is $n$ there is only one $W_{i}$ and the leaves are diffeomorphic to $S^{n-1}$. Otherwise, $L_{i}$ is diffeomorphic to a component of $\Sigma^{+}$or to a component of $\Sigma^{-}$, depending whether $W_{i}$ is a component of $q(t)>0$ or a component of $q(t)<0$. We have the following

Proposition 10.1. For $q \geq 1$ we have

$$
H_{\omega}^{q}\left(C_{0}\left(W_{i}\right)\right) \cong H^{q-1}\left(L_{i}\right) \otimes \mathcal{S} .
$$


Proof. Since the proof is similar in all cases, we assume that $\nu \neq|n-2|$ and $W_{i}=\mathbb{R}_{+}^{n+1}$. In this case

$$
L_{i} \cong S^{k-1} \times B^{n-k} .
$$

The idea of the proof is to construct a manifold $M$ such that

$$
\begin{gathered}
D: M \times(-\epsilon, \epsilon) \times(0, \delta) \longrightarrow W_{i} \quad \text { is a diffeomorphism; } \\
H^{q}(M)=H^{q}\left(L_{i}\right) \quad \forall q \\
D^{*} \omega=d(x+i y) ; \text { and } \\
H_{\omega}^{q}\left(C_{0}\left(W_{i}\right)\right) \cong H_{d(x+i y)}^{q}(\mathcal{F}(y=0))
\end{gathered}
$$

Proposition 1.1 would then give the desired result.

First we construct a special map in the plane that sends

$$
([0,1] \times\{0\}) \cup(\{1\} \times[0, \epsilon]) \quad \text { onto } \quad[0,1+\epsilon] \times\{0\}
$$

and has additional properties which we list later. Consider the $C^{\infty}$ function $\chi(\rho)$ of the single variable $\rho$ defined by

$$
\begin{gathered}
\chi(\rho)=1 \quad \text { for } \rho \leq \frac{1}{3} ; \\
\chi(\rho)=0 \text { for } \rho \geq \frac{2}{3} ; \\
0 \leq \chi(\rho) \leq 1 \quad \text { and }\left|\chi^{\prime}(\rho)\right|<1 \quad \forall \rho .
\end{gathered}
$$

Define the $C^{\infty}$ function $u(\rho, r)$ in the rectangle $0 \leq \rho \leq 1,0 \leq r \leq \frac{1}{2}$ by

$$
u(\rho, r)=\rho \chi(\rho)+\left(\frac{\rho-\frac{2}{3}}{1-r}+\frac{2}{3}\right)(1-\chi(\rho)) .
$$

Notice that

$$
u(\rho, 0)=\rho \quad \forall \rho \in[0,1] .
$$

Let $\delta$ and $\epsilon$ be small positive numbers and let $O(\delta, \epsilon)$ be the closed set in $[0,1] \times\left[0, \frac{1}{2}\right]$ bounded by the curves

$$
\rho=0, r=0, \rho=1, r^{2}\left(1-\rho^{2}\right)=\delta, \text { and } \frac{\rho-\frac{2}{3}}{1-r}+\frac{2}{3}=1+\epsilon .
$$

For $u(\rho, r)$ as in (10.4), consider the $C^{\infty}$ map

$$
\begin{gathered}
\psi_{\delta, \epsilon}: O(\delta, \epsilon) \longrightarrow[0,1+\epsilon] \times[0, \delta] ; \\
\psi_{\delta, \epsilon}(\rho, r)=(u, y) \quad \text { with }\left\{\begin{array}{l}
u=u(\rho, r), \\
y=r^{2}\left(1-\rho^{2}\right) .
\end{array}\right.
\end{gathered}
$$


It is easy to verifiy that
(a) $\psi_{\delta, \epsilon}$ is a homeomorphism;
(b) $\psi_{\delta, \epsilon}(\rho, r)=\left(\rho, r^{2}\left(1-\rho^{2}\right)\right) \quad$ when $\rho \leq \frac{1}{3}$;
(c) $\psi_{\delta, \epsilon}(\rho, 0)=(\rho, 0)$;
(d) $\psi_{\delta, \epsilon}(1, r)=\left(\frac{3-2 r}{3(1-r)}, 0\right)$;
(e) $\psi_{\delta, \epsilon}(0, r)=\left(0, r^{2}\right)$;
(f) $\psi_{\delta, \epsilon}:$ Interior $(O(\delta, \epsilon)) \longrightarrow(0,1+\epsilon) \times(0, \delta)$ is a diffeomorphism.

Furthermore the map $\psi_{\delta, \epsilon}{ }^{-1}$ extends as a $C^{\infty}$ map on the side $y=0$, except at the point $(u=1, y=0)$.

Now we construct a sequence of maps that will define the manifold $M$. Let

$$
\Pi_{1}: \mathbb{R} \times \overline{\mathbb{R}^{+}} \times S^{n-1} \longrightarrow \mathbb{R} \times \mathbb{R}^{n} ; \quad \Pi_{1}(x, r, \theta)=(x, r \theta) .
$$

We have

$$
\overline{\Pi_{1}^{-1}\left(W_{i}\right)}=\mathbb{R} \times \overline{\mathbb{R}^{+}} \times \overline{\Sigma^{+}} .
$$

Let $\phi^{+}$be the diffeomorphism defined in (7.20) and let

$$
\begin{gathered}
\Phi^{+}: \mathbb{R} \times \overline{\mathbb{R}^{+}} \times S^{k-1} \times \overline{B^{n-k}} \longrightarrow \mathbb{R} \times \overline{\mathbb{R}^{+}} \times \overline{\Sigma^{+}} \\
\Phi^{+}(x, r, \theta, \tau)=\left(x, r, \phi^{+}(\theta, \tau)\right)=\left(x, r, \frac{1}{\sqrt{2}} \sqrt{2-|\tau|^{2}} \theta, \frac{1}{\sqrt{2}} \tau\right) .
\end{gathered}
$$

We have

$$
\left(\Phi^{+}\right)^{*}\left(\Pi_{1}^{*} d(x+i q(t))\right)=d\left(x+i r^{2}\left(1-|\tau|^{2}\right)\right) .
$$

Let

$$
c: S^{n-k-1} \times[0,1] \longrightarrow \overline{B^{n-k}} ; c(\alpha, \rho)=\rho \alpha
$$

and let

$$
\begin{gathered}
C: \mathbb{R} \times S^{k-1} \times S^{n-k-1} \times[0,1] \times \overline{\mathbb{R}^{+}} \longrightarrow \mathbb{R} \times S^{k-1} \times \overline{B^{n-k}} \times \overline{\mathbb{R}^{+}} \\
C(x, \theta, \alpha, \rho, r)=(x, \theta, c(\alpha, \rho), r)=(x, \theta, \rho \alpha, r) .
\end{gathered}
$$

Note that

$$
C^{*}\left(\left(\Phi^{+}\right)^{*}\left(\Pi_{1}^{*} d(x+i q(t))\right)\right)=d\left(x+i r^{2}\left(1-\rho^{2}\right)\right)
$$

does not depend on $\alpha$ and $\theta$. For $O(\delta, \epsilon)$ and $\psi_{\delta, \epsilon}$ as above, define

$\Psi_{\delta, \epsilon}: \mathbb{R} \times S^{k-1} \times S^{n-k-1} \times O(\delta, \epsilon) \longrightarrow \mathbb{R} \times S^{k-1} \times S^{n-k-1} \times[0,1+\epsilon] \times[0, \delta] ;$

$$
\Psi_{\delta, \epsilon}(x, \theta, \alpha,(\rho, r))=\left(x, \theta, \alpha, \psi_{\delta, \epsilon}(\rho, r)\right) .
$$

Thus,

$$
\Psi_{\delta, \epsilon}^{*} d\left(x+i r^{2}\left(1-\rho^{2}\right)\right)=d(x+i y)
$$

Let

$$
c_{1+\epsilon}: S^{n-k-1} \times[0,1+\epsilon] \longrightarrow \overline{B^{n-k}}(1+\epsilon) ; \quad c_{1+\epsilon}(\alpha, \rho)=\rho \alpha,
$$


where $B^{n-k}(1+\epsilon)$ is the ball with radius $1+\epsilon$ in $\mathbb{R}^{n+1}$, and let

$$
\begin{gathered}
F_{\delta, \epsilon}: \mathbb{R} \times S^{k-1} \times S^{n-k-1} \times[0,1+\epsilon] \times[0, \delta] \\
\longrightarrow \mathbb{R} \times S^{k-1} \times \overline{B^{n-k}}(1+\epsilon) \times[0, \delta] \\
F_{\delta, \epsilon}(x, \theta, \alpha, u, y)=\left(x, \theta, c_{1+\epsilon}(\alpha, u), y\right) .
\end{gathered}
$$

Now we define the manifold $M$ as

$$
M=S^{k-1} \times B^{n-k}(1+\epsilon)
$$

and the diffeomorphism $D$ as

$$
D=\Pi_{1} \circ \Phi^{+} \circ C \circ \Psi_{\delta, \epsilon}^{-1} \circ F_{\delta, \epsilon}^{-1} .
$$

Then,

$$
H^{q}(M) \cong H^{q}\left(S^{k-1}\right) \cong H^{q}\left(L_{i}\right)
$$

and all the conditions stated at the begining of the proof hold. Let $Y$ be the submanifold of $M \times \mathbb{R} \times[0, \delta]$ given by $y=0$, i.e.,

$$
Y=\{(p, x, y) \in M \times \mathbb{R} \times[0, \delta] ; y=0\} .
$$

Now, it is not difficult to see that if $f \in C_{0}\left(W_{i}\right)$, then $f \circ D \in \mathcal{F}(Y)$ and if $g \in \mathcal{F}(Y)$, then $g \circ D^{-1}$ extends (by 0) as an element of $C_{0}\left(W_{i}\right)$. It follows from all of this that

$$
H_{\omega}^{q}\left(C_{0}\left(W_{i}\right)\right) \cong H_{d(x+i y)}^{q}(\mathcal{F}(Y)) \cong H^{q-1}\left(S^{k-1}\right) \otimes \mathcal{S} .
$$

The proposition is therefore proved in this case.

When $W_{i}=\mathbb{R}_{-}^{n+1}$, a similar argument would give

$$
H_{\omega}^{q}\left(C_{0}\left(W_{i}\right)\right) \cong H^{q-1}\left(S^{n-k-1}\right) \otimes \mathcal{S}^{\prime},
$$

where the space $\mathcal{S}^{\prime}$ is defined in an analogous way as $\mathcal{S}$ (replace $y \geq 0$ in the definition of $\mathcal{S}$ by $y \leq 0$ ). But since $\mathcal{S}^{\prime} \sim \mathcal{S}$, then $\mathcal{S}^{\prime}$ can be replaced by $\mathcal{S}$ in this last isomorphism.

We conclude this section by proving the following proposition and a remark about a canonical representation of 1-cocycles.

Proposition 10.2. Let $\eta \in Z^{q}{ }_{\omega}\left(C_{0}\left(W_{i}\right)\right)$ with $q>1$. If there exists $\mu \in \Omega^{q-1}\left(\mathcal{E}^{n+1}\right)$ such that

$$
\eta=d \mu \quad \bmod (\omega) \quad \text { in } W_{i},
$$

then $\mu \in \Omega^{q-1}\left(\mathcal{F}_{0}(\{q(t)=0\})\right)$.

Proof. Let $M$ and $D$ be as defined in (10.18) and (10.19). Let

$$
\widetilde{\eta}=D^{*} \eta \quad \text { and } \quad \tilde{\mu}=D^{*} \mu .
$$

As noticed above $\widetilde{\eta}$ is flat along $Y$. Now $D$ is $C^{\infty}$ except at the points $(x, \theta, u \alpha, y)$ for which $u=1$. In particular $\widetilde{\mu}$ is $C^{\infty}$ in a neighborhood of

$$
\{0\} \times S^{k-1} \times B^{n-k} \times\{0\} \subset \mathbb{R} \times S^{k-1} \times B^{n-k} \times[0, \delta] .
$$

Next, if $\theta$ is a generator of $H^{q-1}\left(S^{k-1} \times B^{n-k}\right)$, then it follows from (3.14) that

$$
\widetilde{\eta}=f(x, y) \theta \wedge d(x-i y)+d \mu_{0} \bmod (d(x+i y)),
$$


with $f$ flat along $y=0$ and $\mu_{0}$ a $(q-1)$-form flat along $y=0$. If $j_{x_{0}}$ is the embedding defined in (1.9), then $D \circ j_{x_{0}}(p)=\left(x_{0}, 0\right)$ is a constant map. Thus

$$
j_{x_{0}}^{*} \tilde{\mu}=\left(D \circ j_{x_{0}}\right)^{*} \mu=0 \quad \forall x_{0}
$$

since $\mu$ is a $(q-1)$-form with $q-1>0$. Therefore, it follows from Proposition 1.2 that $f=0$ and $\widetilde{\mu}$ is flat along $y=0$. Consequently $\mu$ is flat along $q(t)=0$. This completes the proof.

Remark 10.1. For $\eta \in Z_{\omega}^{1}\left(\mathcal{F}_{0}(\{q(t)=0\})\right)$, let $\eta_{i}$ be its restriction to $W_{i}$. Expression (10.24) is still valid in this case $(q=1)$. This means that $\eta$ is $\omega$-cohomologous to a form $\eta_{0}$ defined by

$$
\eta_{0}=f_{i}(x, q(t)) d \bar{z}_{i}(x, q(t)) \quad \text { in } W_{i},
$$

where $z_{i}$ is a first integral of $\omega$ in $W_{i}$ and the function $f_{i}(x, s)$ is flat along the $x$-axis $s=0$.

\section{High ORDER COHOMOLOGY GROUPS}

We characterize here the cohomology groups $H_{\omega}^{q}$ when $q>1$.

Theorem 11.1. Let $\omega$ be the germ at $0 \in \mathbb{R}^{n+1}$ of a Mizohata structure with signature $\nu$. Then for $q>1$ we have

$$
\begin{array}{ll}
H_{\omega}^{q}\left(\mathcal{E}^{n+1}\right) \cong H^{q-1}\left(S^{k-1} \times S^{n-k-1}\right) \otimes \mathcal{S} & \text { if } \nu \neq n, \\
H_{\omega}^{q}\left(\mathcal{E}^{n+1}\right) \cong H^{q-1}\left(S^{n-1}\right) \otimes \mathcal{S} & \text { if } \nu=n .
\end{array}
$$

Proof. Let $\eta \in Z^{q}{ }_{\omega}\left(\mathcal{E}^{n+1}\right)$. From Proposition 9.1, we can write

$$
\eta=d \mu+\eta^{0} \quad \bmod (\omega)
$$

where $\mu \in \Omega^{q-1}\left(\mathcal{E}^{n+1}\right)$ and $\eta^{0} \in Z^{q}{ }_{\omega}\left(\mathcal{F}_{0}(\{q(t)=0\})\right)$. Define

$$
\eta_{i}^{0}= \begin{cases}\eta^{0} & \text { in } W_{i}, \\ 0 & \text { in } \mathbb{R}^{n+1} \backslash W_{i},\end{cases}
$$

where, as in section 10 , the $W_{i}$ 's denote the components of $\mathbb{R}^{n+1} \backslash \mathbb{R} \times V_{0}$. We have then

$$
\eta^{0}=\sum_{i} \eta_{i}^{0}
$$

It follows from Proposition 10.2 that

$$
\eta_{i}^{0} \in B_{\omega}^{q}\left(\mathcal{E}^{n+1}\right) \Longrightarrow \eta_{i}^{0} \in B_{\omega}^{q}\left(C_{0}\left(W_{i}\right)\right) .
$$

Now, it is easy to see that the correspondence

$$
\begin{aligned}
H_{\omega}^{q}\left(\mathcal{E}^{n+1}\right) & \longrightarrow \bigoplus_{i} H_{\omega}^{q}\left(C_{0}\left(W_{i}\right)\right) \\
{[\eta] } & \longrightarrow \sum_{i}\left[\eta_{i}^{0}\right]
\end{aligned}
$$

is an isomorphism. This together with 10.1 gives

$$
H_{\omega}^{q}\left(\mathcal{E}^{n+1}\right) \cong \bigoplus_{i}\left(H^{q-1}\left(L_{i}\right) \otimes \mathcal{S}\right)
$$


Finally, since the $L_{i}$ 's are diffeomorphic to the components of

$$
\Sigma^{+}=S^{k-1} \times B^{n-k} \quad \text { and } \quad \Sigma^{-}=B^{k} \times S^{n-k-1},
$$

when $\nu \neq n$, and to $S^{n-1}$ when $\nu=n,(11.1)$ and (11.2) follow.

\section{The FIRST COHOMOLOGY GROUP IN DIMENSION TWO}

We construct an isomorphism between the first cohomology group relative to a Mizohata structure in the plane and a space $\mathcal{Y}_{l}$ defined below.

Recall that $\mathcal{A}_{+}\left(\operatorname{resp} \mathcal{A}_{-}\right)$denotes the space of germ at $0 \in \mathbb{R}$ of $C^{\infty}$ functions that have a holomorphic extension to the upper (resp. lower) half plane. For $l \in C^{\infty}$ Dif $f_{0}^{+}$, we define an equivalence relation $\sim_{l}$ on $\mathcal{A}_{-} \times \mathcal{A}_{-}$by

$$
(f, g) \sim_{l}\left(f^{\prime}, g^{\prime}\right) \Longleftrightarrow\left(\exists h, k \in \mathcal{A}_{+} ;\left(f-f^{\prime}\right) \circ l+h \circ l=\left(g-g^{\prime}\right)+k\right) .
$$

Denote by $\mathcal{Y}_{l}$ the quotient space and by $[f, g]_{l}$ the class of $(f, g)$. The following lemma says that $\mathcal{Y}_{l}$ depends only on the class $[l]$ of $l$ in $\mathcal{D}_{0}^{+}$(see section 6 ).

Lemma 12.1. If $l^{\prime} \in C^{\infty}$ Diff $f_{0}^{+}$is such that $\left[l^{\prime}\right]=[l]$ in $\mathcal{D}_{0}^{+}$, then

$$
\mathcal{Y}_{l^{\prime}} \cong \mathcal{Y}_{l} \text {. }
$$

Proof. If $\left[l^{\prime}\right]=[l]$, then there exist $a, b, \in C^{\varpi}$ Dif $f_{0}^{+}$such that $l^{\prime}=a \circ l \circ b$. The automorphism

$$
\Phi_{a, b}: \mathcal{A}_{-} \times \mathcal{A}_{-} \longrightarrow \mathcal{A}_{-} \times \mathcal{A}_{-} ; \Phi_{a, b}(f, g)=\left(f \circ a^{-1}, g \circ b\right)
$$

is then easily seen to satisfy

$$
(f, g) \sim_{l}\left(f^{\prime}, g^{\prime}\right) \Longleftrightarrow \Phi_{a, b}(f, g) \sim_{l^{\prime}} \Phi_{a, b}\left(f^{\prime}, g^{\prime}\right) .
$$

It therefore induces an isomorphism from $\mathcal{Y}_{l}$ into $\mathcal{Y}_{l^{\prime}}$

Let $\omega_{\rho}$ be as in (6.1) and $z^{ \pm}$be its (semi) first integrals defined in section 6 . Recall that the class in $\mathcal{D}_{0}^{+}$of

$$
l(u)=z^{+} \circ\left(z^{-}\right)^{-1}(u)=z^{+}(u), \quad u \in \mathbb{R},
$$

is a representative of $\omega_{\rho}$. Now we can state

Theorem 12.1. For $\omega_{\rho}$ and $l$ as above, we have

$$
H_{\omega_{\rho}}^{1}\left(\mathcal{E}^{2}\right) \cong \mathcal{Y}_{l}
$$

The remainder of this section is devoted to the construction of the isomorphism (12.5). Let

$$
\eta=a(x, t) d x+b(x, t) d t \in \Omega^{1}\left(\mathcal{E}^{2}\right) .
$$

Without loss of generality (Proposition 8.1), we can assume that $a, b \in \mathcal{F}_{0}(\{t=0\})$. The equation

$$
d u \wedge \omega_{\rho}=\eta \wedge \omega_{\rho}
$$

is equivalent to

$$
L_{\rho} u=f,
$$

where

$$
L_{\rho}=\frac{\partial}{\partial t}-2 i t(1+\rho) \frac{\partial}{\partial x} \quad \text { and } \quad f=2 i t(1+\rho) a-b .
$$


The pushforwards via $z^{ \pm}$of (12.8) in rectangles $R^{ \pm}(\epsilon, \delta)$ (see section 6) are the equations

$$
\frac{\partial \widetilde{u}^{ \pm}}{\partial \bar{\zeta}}(\zeta)=\frac{\widetilde{f^{ \pm}}}{\widetilde{L_{\rho} \bar{z}^{ \pm}}}(\zeta)=\phi^{ \pm}(\zeta) \quad \text { in } z^{ \pm}\left(R^{ \pm}(\epsilon, \delta)\right) \subset \mathbb{C},
$$

where for a function $A(x, t)$ defined in the $(x, t)$-plane $\widetilde{A}^{ \pm}$means

$$
\widetilde{A}^{ \pm}(\zeta)=A \circ\left(z^{ \pm}\right)^{-1}(\zeta) \quad \text { in } z^{ \pm}\left(R^{ \pm}(\epsilon, \delta)\right) .
$$

Since our problem is local and $f$ is flat along $t=0$, then we can assume that $\tilde{f}^{ \pm}$is defined in $\mathbb{C}$ and compactly supported in $z^{ \pm}\left(R^{ \pm}(\epsilon, \delta)\right)$. The $C^{\infty}$ functions

$$
\widetilde{\phi}_{0}^{ \pm}(\zeta)=\frac{1}{2 \pi i} \int_{\mathbb{C}} \frac{\phi^{ \pm}(\mu)}{\zeta-\mu} d \mu \wedge d \bar{\mu}
$$

satisfy then

$$
\frac{\partial \widetilde{u}_{0}^{ \pm}}{\partial \bar{\zeta}}=\phi^{ \pm}
$$

In particular $\widetilde{u}_{0}^{ \pm}$are holomorphic in the lower half plane $\Im \zeta<0$. Denote by $v^{ \pm}$the restriction of $\widetilde{u}_{0}^{ \pm}$to $\Im \zeta=0$. We have then (in terms of germs) $v^{ \pm} \in \mathcal{A}_{-}$. Set

$$
I_{l}: \Omega^{1}\left(\mathcal{F}_{0}(\{t=0\})\right) \longrightarrow \mathcal{Y}_{l} ; \quad I_{l}(\eta)=\left[v^{+}, v^{-}\right]_{l} .
$$

Lemma 12.2. If $\eta$ is $\omega_{\rho}$-exact, then $I_{l}(\eta)=0$.

Proof. Let $\psi$ be a $C^{\infty}$ solution of (12.7) and let $\psi^{ \pm}$be the restriction of $\psi$ to $R^{ \pm}(\epsilon, \delta)$. We have then

$$
d\left(\psi^{ \pm}-\widetilde{u}_{0}^{ \pm} \circ z^{ \pm}\right) \wedge d z^{ \pm}=0 \quad \text { in } R^{ \pm}(\epsilon, \delta) .
$$

Therefore,

$$
\psi^{ \pm}-\widetilde{u}_{0}^{ \pm} \circ z^{ \pm}=h^{ \pm} \circ z^{ \pm} \quad \text { in } R^{ \pm}(\epsilon, \delta),
$$

where $h^{ \pm}$is holomorphic in the interior of $z^{ \pm}\left(R^{ \pm}(\epsilon, \delta)\right)$ and is $C^{\infty}$ up to the boundary. Since

$$
\psi^{+}(x, 0)=\psi^{-}(x, 0)
$$

then

$$
\widetilde{u}_{0}^{+} \circ z^{+}(x, 0)+h^{+} \circ z^{+}(x, 0)=\widetilde{u}_{0}^{-} \circ z^{-}(x, 0)+h^{-} \circ z^{-}(x, 0),
$$

or equivalently

$$
v^{+} \circ l+h^{+} \circ l=v^{-}+h^{-}
$$

which means $\left[v^{+}, v^{-}\right]_{l}=0$.

This lemma shows that $I_{l}$ induces a homomorphism (that we will call again $I_{l}$ )

$$
I_{l}: H_{\omega_{\rho}}^{1}\left(\mathcal{E}^{2}\right) \longrightarrow \mathcal{Y}_{l} ; \quad I_{l}[\eta]=I_{l}(\eta) .
$$

Now we prove that $I_{l}$ is the sought isomorphism of Theorem 12.1 . 
Surjectivity of $I_{l}$. Let $[f, g]_{l} \in \mathcal{Y}_{l}$ with $f, g \in \mathcal{A}_{-}$. Let $F, G$ be extensions of $f, g$ to a neighborhood of $0 \in \mathbb{C}$ which are holomorphic in $\Im \zeta<0$. Define a function $m(x, t)$ in a neighborhood of $0 \in \mathbb{R}^{2}$ by

$$
m(x, t)= \begin{cases}\frac{\partial F}{\partial \bar{\zeta}}\left(z^{+}(x, t)\right), & \text { in } R^{+}(\epsilon, \delta), \\ \frac{\partial G}{\partial \bar{\zeta}}\left(z^{-}(x, t)\right), & \text { in } R^{-}(\epsilon, \delta) .\end{cases}
$$

Clearly $m$ is flat along $t=0$. Define a 1 -form $\eta$ by

$$
\eta=-\frac{m(x, t)}{z_{x}^{ \pm}(x, t)} L_{\rho} \overline{z^{ \pm}}(x, t) d t, \quad \text { in } R^{ \pm}(\epsilon, \delta) .
$$

It is easily verified that the equation $(\eta-d u) \wedge \omega_{\rho}=0$ gives rise to

$$
\frac{\partial \widetilde{u}^{+}}{\partial \bar{\zeta}}=\frac{\partial F}{\partial \bar{\zeta}} \quad \text { and } \quad \frac{\partial \widetilde{u}^{-}}{\partial \bar{\zeta}}=\frac{\partial G}{\partial \bar{\zeta}} .
$$

Therefore, $I_{l}(\eta)=[f, g]_{l}$.

Injectivity of $I_{l}$. Suppose that $I_{l}(\eta)=\left[v^{+}, v^{-}\right]_{l}=0$. Then there exist $h^{ \pm} \in \mathcal{A}_{+}$ such that

$$
v^{+} \circ l+h^{+} \circ l=v^{-}+h^{-} .
$$

Define a function $u(x, t)$ by

$$
u(x, t)=\widetilde{u}_{0}^{ \pm}\left(z^{ \pm}(x, t)\right)+h^{ \pm}\left(z^{ \pm}(x, t)\right) \quad \text { in } R^{ \pm}(\epsilon, \delta) .
$$

It follows from (12.20) and from $z_{t}^{ \pm}(x, 0)=0$ that $u$ is a $C^{1}$ function. Easily,

$$
\eta \wedge \omega_{\rho}=d u \wedge \omega_{\rho}
$$

To finish the proof, we need only to show that $u \in C^{\infty}$. We rewrite (12.22) as

$$
u_{t}=2 i t(1+\rho) u_{x}+f \text {. }
$$

Since the restriction $u^{+}$(resp. $u^{-}$) of $u$ to $t>0$ (resp. $t<0$ ) are $C^{\infty}$ and since all its partial derivatives extend as continuous functions to $t \leq 0$ (resp. $t \geq 0$ ), then we need only to show that the partial derivatives of $u^{+}$agree with those of $u^{-}$on $t=0$. For this, we can use an argument similar to that used by Treves to prove Lemma VII 3.1 in [Tr4]. More precisely, since $\rho$ and $f$ are flat along $t=0$, then we can prove (by induction) that the partial derivatives of $u^{ \pm}$on $t=0$ are fully determined by the derivatives of its trace $u(x, 0)$. This proves the injectivity of $I_{l}$ and also the theorem.

\section{THE FIRST COHOMOLOGY GROUP WHEN $\nu=n-2$ AND $n>2$}

In this section we will prove that the the first cohomology group relative to a Mizohata structure with signature $n-2, n>2$, and with representative $[l]$ is isomorphic to the space $\mathcal{Z}_{l}$ constructed below.

For $l \in C^{\infty}$ Diff $f_{0}^{+}$, consider the equivalence relation $\sim_{l}$ in $\mathcal{A}_{-} \times \mathcal{A}_{-} \times \mathcal{A}_{+}$by

$$
(\alpha, \beta, \gamma) \sim_{l}\left(\alpha^{\prime}, \beta^{\prime}, \gamma^{\prime}\right) \Longleftrightarrow\left\{\begin{aligned}
\exists(h, k, m) \in \mathcal{A}_{+} & \times \mathcal{A}_{+} \times \mathcal{A}_{-} ; \\
\left(\alpha-\alpha^{\prime}\right) \circ l+h \circ l & =\left(\beta-\beta^{\prime}\right)+k \\
& =\left(\gamma-\gamma^{\prime}\right)+m .
\end{aligned}\right.
$$

Denote by $\mathcal{Z}_{l}$ the quotient space and by $[\alpha, \beta, \gamma]_{l}$ the class of $(\alpha, \beta, \gamma)$. 
As in Lemma 12.1, we can easily prove

Lemma 13.1. If $l^{\prime} \in C^{\infty}$ Dif $f_{0}^{+}$satisfies $\left[l^{\prime}\right]=[l] \in \mathcal{D}_{0}^{+}$, then

$$
\mathcal{Z}_{l^{\prime}} \cong \mathcal{Z}_{l}
$$

Let $\omega_{\rho}$ be the germ at $0 \in \mathbb{R}^{n+1}(n>2)$ of a Mizohata structure with signature $n-2$ described in (7.11). Let $\omega_{\rho}^{0}$ be as in (7.12), let $z^{ \pm}(x, s)$ be the (semi) first integrals of $\omega_{\rho}^{0}$ as defined in section 6 , and let

$$
l(u)=z^{+} \circ\left(z^{-}\right)^{-1}(u)=z^{+}(u), \quad u \in \mathbb{R} .
$$

Thus $[l] \in \mathcal{D}_{0}^{+}$is a representative of $\omega_{\rho}^{0}$ and also that of $\omega_{\rho}$. The remaining of this section is devoted to the construction of an isomorphism between $H_{\omega_{\rho}}^{1}\left(\mathcal{E}^{n+1}\right)$ and $\mathcal{Z}_{l}$.

Let $\eta \in Z_{\omega_{\rho}}^{1}\left(\mathcal{E}^{n+1}\right)$. We can assume (thanks to Proposition 9.1) that $\eta \in$ $Z_{\omega_{\rho}}^{1}\left(\mathcal{F}_{0}(\{q(t)=0\})\right)$. It then follows from remark 10.1 that there exist $f^{+,+}$, $f^{+,-}$, and $f^{-}$defined in the $(x, s)$-plane such that

$$
\operatorname{supp} f^{+, \pm} \subset\{s \geq 0\}, \quad \operatorname{supp} f^{-} \subset\{s \leq 0\},
$$

and such that $\eta$ is $\omega_{\rho}$-cohomologous to the form $\eta_{0}$ defined by

$$
\eta_{0}= \begin{cases}f^{+,+}(x, \sqrt{q(t)}) d \overline{z^{+}}(x, q(t)), & \text { in } \mathbb{R}_{+,+}^{n+1}, \\ f^{+,-}(x, \sqrt{q(t)}) d(x-i q(t)), & \text { in } \mathbb{R}_{+,-}^{n+1}, \\ f^{-}(x,-\sqrt{-q(t)}) d(x-i q(t)), & \text { in } \mathbb{R}_{-}^{n+1} .\end{cases}
$$

Making use of (13.6), we solve equation $\left(\eta_{0}-d u\right) \wedge \omega_{\rho}=0$ in $\mathbb{R}_{+, \pm}^{n+1}$ and $\mathbb{R}_{-}^{n+1}$. The traces along the $x$-axis of these solutions generate an element in $\mathcal{Z}_{l}$.

In the $(x, s)$-plane consider the following equations:

$$
\begin{aligned}
d u^{+,+} \wedge d z^{+} & =f^{+,+}(x, s) d \overline{z^{+}} \wedge d z^{+} ; \\
d u^{+,-} \wedge d\left(x+i s^{2}\right) & =f^{+,-}(x, s) d\left(x-i s^{2}\right) \wedge d\left(x+i s^{2}\right) ; \\
d u^{-} \wedge d\left(x-i s^{2}\right) & =f^{-}(x, s) d\left(x+i s^{2}\right) \wedge d\left(x-i s^{2}\right) .
\end{aligned}
$$

We denote by $\widetilde{f}^{+,+}, \widetilde{f}^{+,-}$, and $\widetilde{f}^{-}$the pushforwards via $z^{+}, z^{-}=x+i s^{2}$, and $\bar{z}^{-}=x-i s^{2}$ of $f^{+,+}, f^{+,-}$, and $f^{-}$, respectively. Let

$$
\widetilde{u}_{0}^{+, \pm}(\zeta)=\frac{1}{2 \pi i} \int_{\mathbb{C}} \frac{\widetilde{f}^{+, \pm}(\mu)}{\zeta-\mu} d \mu \wedge d \bar{\mu} \quad \text { and } \quad \widetilde{u}_{0}^{-}(\zeta)=\frac{1}{2 \pi i} \int_{\mathbb{C}} \frac{\widetilde{f}^{-}(\mu)}{\zeta-\mu} d \mu \wedge d \bar{\mu}
$$

Notice that the functions

$$
u^{+, \pm}(x, t)=\widetilde{u}_{0}^{+, \pm}\left(z^{ \pm}(x, q(t))\right) \quad \text { and } \quad u^{-}(x, t)=\widetilde{u}_{0}^{-}(x+i q(t))
$$

satisfy

$$
\begin{aligned}
d u^{+, \pm} \wedge \omega_{\rho} & =\eta_{0} \wedge \omega_{\rho}, & & \text { in } \mathbb{R}_{+, \pm}^{n+1}, \\
d u^{-} \wedge \omega_{\rho} & =\eta_{0} \wedge \omega_{\rho}, & & \text { in } \mathbb{R}_{-}^{n+1} .
\end{aligned}
$$

It follows from (13.5) that $\widetilde{u}_{0}^{+, \pm}$are holomorphic in $\Im \zeta<0$ and $\widetilde{u}_{0}^{-}$is holomorphic in $\Im \zeta>0$. Hence, if

$$
v^{+, \pm}=\left.\widetilde{u}_{0}^{+, \pm}\right|_{\Im \zeta=0} \quad \text { and } \quad v^{-}=\left.\widetilde{u}_{0}^{-}\right|_{\Im \zeta=0},
$$


then $\left(v^{+,+}, v^{+,-}, v^{-}\right) \in \mathcal{A}_{-} \times \mathcal{A}_{-} \times \mathcal{A}_{+}$. In this way we can associate with the $\omega_{\rho}$-closed 1 -form $\eta$ an element of $\mathcal{Z}_{l}$, namely

$$
I_{l}(\eta)=\left[v^{+,+}, v^{+,-}, v^{-}\right]_{l} .
$$

The following lemma says that $I_{l}(\eta)$ depends only on the cohomology class of $\eta$.

Lemma 13.2. If $\eta \in B_{\omega_{\rho}}^{1}\left(\mathcal{E}^{n+1}\right)$, then $I_{l}(\eta)=0$.

Proof. Let $\phi \in \mathcal{E}^{n+1}$ be such that

$$
d \phi \wedge \omega_{\rho}=\eta_{0} \wedge \omega_{\rho}
$$

where $\eta_{0}$ is defined in (13.6). This together with (13.11) gives

$$
\begin{aligned}
d\left(\phi-u^{+, \pm}\right) & \wedge \omega_{\rho}=0, & & \text { in } \mathbb{R}_{+, \pm}^{n+1}, \\
d\left(\phi-u^{-}\right) & \wedge \omega_{\rho}=0, & & \text { in } \mathbb{R}_{-}^{n+1} .
\end{aligned}
$$

Thus,

$$
\phi-\widetilde{u}_{0}^{+, \pm} \circ z^{ \pm}=h^{+, \pm} \circ z^{ \pm} \text {and } \phi-\widetilde{u}_{0}^{-} \circ \bar{z}^{-}=h^{-} \circ \bar{z}^{-},
$$

where the smooth functions $h^{+, \pm}$and $h^{-}$, defined near $0 \in \mathbb{C}$, are holomorphic in $\Im \zeta>0$ and $\Im \zeta<0$, respectively, from which it follows that

$$
v^{+,+} \circ l+h^{+,+} \circ l=v^{+,-}+h^{+,-}=v^{-}+h^{-} .
$$

This means $\left[v^{+,+}, v^{+,-}, v^{-}\right]_{l}=0$.

Hence $I_{l}$ induces a homomorphism (that we label again $I_{l}$ ) from $H_{\omega_{\rho}}^{1}\left(\mathcal{E}^{n+1}\right)$ into $\mathcal{Z}_{l}$. Now we prove the bijectivity of this induced homomorphism.

Surjectivity of $I_{l}$. For $f, g \in \mathcal{A}_{-}, m \in \mathcal{A}_{+}$, let $F, G$ and $M$ be smooth extensions of $f, g$ and $m$, respectively, to a full neighborhood of $0 \in \mathbb{C}$ with $F, G$ holomorphic in the lower and $M$ in the upper half planes. It is verified at once that the form $\eta \in Z_{\omega_{\rho}}^{1}\left(\mathcal{F}_{0}(\{q(t)=0\})\right)$ defined by

$$
\begin{array}{ll}
\eta(x, t)=\frac{\partial F}{\partial \bar{\zeta}}\left(z^{+}(x, q(t))\right) d \bar{z}^{+}(x, q(t)), & \text { in } \mathbb{R}_{+,+}^{n+1}, \\
\eta(x, t)=\frac{\partial G}{\partial \bar{\zeta}}(x+i q(t)) d(x-i q(t)), & \text { in } \mathbb{R}_{+,-}^{n+1}, \\
\eta(x, t)=\frac{\partial M}{\partial \bar{\zeta}}(x+i q(t)) d(x-i q(t)), & \text { in } \mathbb{R}_{-}^{n+1}
\end{array}
$$

satisfies $I_{l}[\eta]=[f, g, m]_{l}$.

Injectivity of $I_{l}$. Suppose that $I_{l}[\eta]=0$. Then there exist $h^{+, \pm} \in \mathcal{A}_{+}$and $h^{-} \in \mathcal{A}_{-}$ such that

$$
v^{+,+} \circ l+h^{+,+} \circ l=v^{+,-}+h^{+,-}=v^{-}+h^{-} .
$$

As in the proof of the injectivity of $I_{l}$ when $n=1$ (section 12), it can be shown that the function $u(x, t)$ defined near $0 \in \mathbb{R}^{n+1}$ by

$$
\begin{aligned}
& u(x, t)=\widetilde{u}_{0}^{+, \pm}\left(z^{ \pm}(x, t)\right)+h^{+, \pm}\left(z^{ \pm}(x, t)\right), \quad \text { in } \mathbb{R}_{+, \pm}^{n+1}, \\
& u(x, t)=\widetilde{u}_{0}^{-}\left(z^{-}(x, t)\right)+h^{-}\left(z^{-}(x, t)\right), \quad \text { in } \mathbb{R}_{-}^{n+1}
\end{aligned}
$$

is a $C^{\infty}$ solution of $(d u-\eta) \wedge \omega_{\rho}=0$.

We have then proved the following theorem. 
Theorem 13.1. Let $\omega$ be the germ at $0 \in \mathbb{R}^{n+1}$ of a Mizohata structure with signature $n-2$. If $n>2$ and $[l] \in \mathcal{D}_{0}^{+}$is a representative of $\omega$, then

$$
H_{\omega}^{1}\left(\mathcal{E}^{n+1}\right) \cong \mathcal{Z}_{l}
$$

\section{The FIRST COHOMOLOGY GROUP WHEN $\nu=0$ AND $n=2$}

As in the last two sections, we construct a space $\mathcal{W}_{l_{1}, l_{2}}$ from $\mathcal{A}_{ \pm}$and then show that this new space is isomorphic to $H_{\omega_{\rho_{1}, \rho_{2}}}^{1}\left(\mathcal{E}^{3}\right)$, where $\omega_{\rho_{1}, \rho_{2}}$ is the germ of a Mizohata structure at $0 \in \mathbb{R}^{3}$ with signature 0 and with representative $\left[l_{1}, l_{2}\right]$ in $\mathcal{E}_{0}^{+}$. by

For $l_{1}, l_{2} \in C^{\infty}$ Diff $f_{0}^{+}$, we define an equivalence relation in $\mathcal{A}_{-} \times \mathcal{A}_{-} \times \mathcal{A}_{+} \times \mathcal{A}_{+}$

$$
(\alpha, \beta, \gamma, \delta) \sim_{l_{1}, l_{2}}\left(\alpha^{\prime}, \beta^{\prime}, \gamma^{\prime}, \delta^{\prime}\right) \Longleftrightarrow\left\{\begin{aligned}
\exists(h, k, m, n) \in \mathcal{A}_{+} & \times \mathcal{A}_{+} \times \mathcal{A}_{-} \times \mathcal{A}_{-} ; \\
\left(\alpha-\alpha^{\prime}\right) \circ l_{1}+h \circ l_{1} & =\left(\beta-\beta^{\prime}\right)+k \\
& =\left(\gamma-\gamma^{\prime}\right) \circ l_{2}+m \circ l_{2} \\
& =\left(\delta-\delta^{\prime}\right)+n .
\end{aligned}\right.
$$

Denote by $\mathcal{W}_{l_{1}, l_{2}}$ the quotient space and by $[\alpha, \beta, \gamma, \delta]_{l_{1}, l_{2}}$ the class of $(\alpha, \beta, \gamma, \delta)$. The following lemma says that $\mathcal{W}_{l_{1}, l_{2}}$ depends only on the class of $\left(l_{1}, l_{2}\right)$ in $\mathcal{E}_{0}^{+}$.

Lemma 14.1. Let $l_{1}^{\prime}, l_{2}^{\prime} \in C^{\infty}$ Diff $f_{0}^{+}$be such that $\left[l_{1}^{\prime}, l_{2}^{\prime}\right]=\left[l_{1}, l_{2}\right] \in \mathcal{E}_{0}^{+}$. Then

$$
\mathcal{W}_{l_{1}^{\prime}, l_{2}^{\prime}} \cong \mathcal{W}_{l_{1}, l_{2}} \text {. }
$$

Proof. It follows from the definition of $\mathcal{E}_{0}^{+}$that there exist $a, b, c \in C^{\varpi}$ Diff $f_{0}^{+}$such that

$$
l_{1}^{\prime}=a \circ l_{1} \circ c \text { and } l_{2}^{\prime}=b \circ l_{2} \circ c .
$$

It is now easy to see that the automorphism $\Phi_{a, b, c}$ of $\mathcal{A}_{-} \times \mathcal{A}_{-} \times \mathcal{A}_{+} \times \mathcal{A}_{+}$defined by

$$
\Phi_{a, b, c}(\alpha, \beta, \gamma, \delta)=\left(\alpha \circ a^{-1}, \beta \circ c, \gamma \circ b^{-1}, \delta \circ c\right)
$$

induces the isomorphism (14.2).

Let $\omega=\omega_{\rho_{1}, \rho_{2}}$ be as in (7.13) and $\omega_{\rho_{1}}^{0}, \omega_{\rho_{2}}^{0}$ be as in (7.14). For $j=1,2$, let $z_{j}^{ \pm}(x, s)$ be the (semi) first integrals of $\omega_{\rho_{j}}^{0}$, as defined in section 6 , and let

$$
l_{j}(u)=z_{j}^{+} \circ\left(z_{j}^{-}\right)^{-1}(u)=z_{j}^{+}(u), \quad u \in \mathbb{R} .
$$

Thus, $\left[l_{1}, l_{2}\right] \in \mathcal{E}_{0}^{+}$is the representative of the Mizohata structure defined by $\omega_{\rho_{1}, \rho_{2}}$. We are now going to construct an isomorphism between $H_{\omega_{\rho_{1}, \rho_{2}}}^{1}\left(\mathcal{E}^{3}\right)$ and $\mathcal{W}_{l_{1}, l_{2}}$.

Let $\eta \in Z_{\omega_{\rho_{1}, \rho_{2}}}^{1}\left(\mathcal{E}^{3}\right)$. From Remark 10.1, $\eta$ is $\omega_{\rho_{1}, \rho_{2}}$-cohomologous to a form $\eta_{0} \in Z_{\omega_{\rho_{1}, \rho_{2}}}^{1}\left(\mathcal{F}_{0}(\{q(t)=0\})\right)$ given by

$$
\begin{array}{ll}
\eta_{0}\left(x, t_{1}, t_{2}\right)=f_{1}^{ \pm}(x, q(t)) d \bar{z}_{1}^{ \pm}(x, q(t)), & \text { in } \mathbb{R}_{+, \pm}^{3}, \\
\eta_{0}\left(x, t_{1}, t_{2}\right)=f_{2}^{ \pm}(x, q(t)) d \bar{z}_{2}^{ \pm}(x, q(t)), & \text { in } \mathbb{R}_{-, \pm}^{3},
\end{array}
$$

where $f_{1}^{ \pm}$and $f_{2}^{ \pm}$are $C^{\infty}$ functions defined near 0 in the $(x, s)$-plane and satisfying

$$
\operatorname{supp} f_{1}^{ \pm} \subset\{s \geq 0\} \quad \text { and } \operatorname{supp} f_{2}^{ \pm} \subset\{s \leq 0\} .
$$


Define the smooth functions $\widetilde{u}_{j}^{ \pm}$in $\mathbb{C}$ by

$$
\widetilde{u}_{j}^{ \pm}(\zeta)=\frac{1}{2 \pi i} \int_{\mathbb{C}} \frac{\widetilde{f}_{j}^{ \pm}(\mu)}{\zeta-\mu} d \mu \wedge d \bar{\mu}, \quad j=1,2,
$$

where $\widetilde{f}_{j}^{ \pm}$is the pushforward via $z_{j}^{ \pm}$of $f_{j}^{ \pm}$. Since $\widetilde{u}_{1}^{ \pm}$is holomorphic in $\Im \zeta<0$ and $\widetilde{u}_{2}^{ \pm}$is holomorphic in $\Im \zeta>0$, then the restrictions $v_{j}^{ \pm}$of $\widetilde{u}_{j}^{ \pm}$to the real axis $\Im \zeta=0$ satisfy

$$
\left(v_{1}^{+}, v_{1}^{-}, v_{2}^{+}, v_{2}^{-}\right) \in \mathcal{A}_{-} \times \mathcal{A}_{-} \times \mathcal{A}_{+} \times \mathcal{A}_{+} .
$$

To the 1-form $\eta$ we can then associate

$$
I_{l_{1}, l_{2}}(\eta)=\left[v_{1}^{+}, v_{1}^{-}, v_{2}^{+}, v_{2}^{-}\right]_{l_{1}, l_{2}} \in \mathcal{W}_{l_{1}, l_{2}} .
$$

Lemma 14.2. If $\eta \in B_{\omega_{\rho_{1}, \rho_{2}}}^{1}\left(\mathcal{E}^{3}\right)$, then $I_{l_{1}, l_{2}}(\eta)=0$.

The proof is analogous to that of lemma 13.2 and is left to the reader. Thus, $I_{l_{1}, l_{2}}$ induces a homomorphism from $H_{\omega_{\rho_{1}, \rho_{2}}}^{1}\left(\mathcal{E}^{3}\right)$ into $\mathcal{W}_{l_{1}, l_{2}}$. The verification of the bijectivity of this homomorphism can be handled in a similar fashion as that of $I_{l}$ in section 13. We summarize this in the following theorem.

Theorem 14.1. Let $\omega$ be the germ at $0 \in \mathbb{R}^{3}$ of a Mizohata structure with signature 0 , and let $\left[l_{1}, l_{2}\right]$ be a representative of $\omega$ in $\mathcal{E}_{0}^{+}$. Then,

$$
H_{\omega}^{1}\left(\mathcal{E}^{3}\right) \cong W_{l_{1}, l_{2}}
$$

\section{A remark on Treves' Conjecture}

Let

$$
z(x, t)=x+i \phi(x, t), \quad \phi(0)=0,
$$

be a $C^{\infty}$ function defined near $0 \in \mathbb{R}^{n+1}$. Denote by $L_{p}$ the fiber through the point $p$ of the structure defined by $d z$, i.e.

$$
L_{p}=\{(x, t) ; z(x, t)=z(p)\} .
$$

Treves [Tr1] has proved that when $\phi$ is independent on $x$, the triviality of $H_{d z}^{q}\left(\mathcal{E}^{n+1}\right)$ is equivalent to the triviality of the cohomology groups $H^{q-1}\left(L_{p}\right)$. When $\phi$ depends on $x$, Cordaro and Treves have proved [CT] the necessity part of this condition. The sufficiency part has been conjectured. Guided by the techniques we have used to treat the Mizohata complex, we will attempt here to justify this conjecture, at least when the separatrix of the foliation defined by $d z$ is an analytic variety.

Let $\Sigma$ be the characteristic set of $d z$ in a small neighborhood $U$ of 0 , i.e.

$$
\Sigma=\{(x, t) \in U ; d z \wedge d \bar{z}=0\},
$$

and let $V=z^{-1}(z(\Sigma))$. Suppose that $V$ is the germ of a real analytic variety. Denote by $W_{1}, \cdots, W_{k}$ the components of $U \backslash V$. By shrinking $U$ if necessary, the function $z$ can be thought of as a fibration in each $W_{i}$. Let $L_{i}$ be the generic fiber of $d z$ in $W_{i}$ and let $C_{0}\left(W_{i}\right)$ be the space of germs at 0 of $C^{\infty}$ functions with support 
in $\bar{W}_{i}$ and flat along $V$. By using the desingularization of $V$ and Borel's extension theorem, then for $q>1$ we can expect the following:

$$
\begin{aligned}
H_{d z}^{q}\left(\mathcal{E}^{n+1}\right) & \cong H_{d z}^{q}\left(\mathcal{F}_{0}(V)\right) ; \\
H_{d z}^{q}\left(\mathcal{F}_{0}(V)\right) & \cong \bigoplus_{i} H_{d z}^{q}\left(C_{0}\left(W_{i}\right)\right) ; \\
H_{d z}^{q}\left(C_{0}\left(W_{i}\right)\right) & \cong H^{q-1}\left(L_{i}\right) \otimes \mathcal{S}_{i},
\end{aligned}
$$

where $\mathcal{S}_{i}$ denotes a space of germs of functions equivalent to the space $\mathcal{S}$ of Lemma 1.1. This would then mean that $H_{d z}^{q}=0$ when $H^{q-1}\left(L_{i}\right)=0$ for $i=1, \cdots, k$.

\section{REFERENCES}

[BT] M.S. Baouendi and F. Treves, A local constancy principle for the solutions of certain overdetermined systems of first-order linear partial differential equations, in Math. Analysis and Applications, Advances in Math. Supplementary Studies, vol. 7A, 1981, pp. 245-262. MR 84f:35105

$[\mathrm{CH}]$ P. Cordaro and J. Hounie, On local solvability of underdetermined systems of vector fields, Amer. J. Math. 112 (2) (1990), 243-270. MR 91j:58150

[CT] P. Cordaro and F. Treves, Homology and cohomology in hypo-analytic structures of the hypersurface type, J. Geo. Analysis 1 (1) (1991), 39-70. MR 92h:32034

[GG] M. Golubitsky and V. Guillemin, Stable mappings and their singularities, Graduate Texts in Math., vol. 14, Springer-Verlag, New York, 1973. MR 49:6269

[MT] G. Mendoza and F. Treves, Local solvability in a class of overdetermined systems of linear PDE, Duke Math. J. 63 (2) (1991), 355-377. MR 92i:58177

[Me1] A. Meziani, Topological invariance of the solvability of complex-valued vector fields in the plane, Duke Math. J. 71 (2) (1993), 609-628. MR 94g:35048

[Me2] _ Classification of germs of Mizohata structures, Comm. PDE 20 (1995), 499-539. MR 96b:35029

[NT] L. Nirenberg and F. Treves, Solvability of a first order partial differential equation, Comm. Pure Appl. Math. 16 (1963), 331-351. MR 29:348

[Tr1] F. Treves, Study of a model in the theory of complexes of pseudodifferential operators, Ann. Math. 104 (1976), 269-324. MR 54:14014

[Tr2] On the local integrability and solvability of systems of vector fields, Acta Math. 151 (1983), 1-48. MR 85j:35009

[Tr3] - On the local solvability for top degree forms in hypo-analytic structures, Amer. J. Math. 112 (1990), 403-421. MR 91j:35005

[Tr4] - Hypo-analytic structures: local theory, vol. 40, Princeton University Press, 1991.

Department of Mathematics, Florida International University, Miami, Florida 33199

E-mail address: meziani@servax.fiu.edu 